\title{
Cross-regulation between CDK and MAPK control cellular fate
}

\author{
Eric Durandau, Serge Pelet* \\ Department of Fundamental Microbiology, University of Lausanne, CH-1015 \\ Lausanne, Switzerland \\ * Corresponding author: serge.pelet@unil.ch
}

\section{Keywords}

MAPK signaling, cell cycle, yeast mating, single cell analysis, fluorescent biosensors

\begin{abstract}
Commitment to a new cell cycle is controlled by a number of cellular signals. Mitogen-Activated Protein Kinase pathways, which transduce multiple extracellular cues, have been shown to be interconnected with the cell cycle. Using budding yeast as a model system, we have quantified in hundreds of live single cells the interplay between the MAPK regulating the mating response and the Cyclin-Dependent Kinase controlling cell cycle progression. Different patterns of MAPK activity dynamics could be identified by clustering cells based on their CDK activity, denoting the tight relationship between these two cellular signals. In mating mixtures, we have verified that the interplay between CDK and MAPK activities allows cells to select their fate, preventing them from being blocked in an undesirable cellular program.
\end{abstract}




\section{Introduction}

2 Cells have developed complex signal transduction pathways to respond to changes in their environment. Plasma membrane sensors detect extracellular stimuli and relay this information inside the cell via signaling cascades. These protein networks can integrate multiple information to launch the appropriate cellular response. For instance, Mitogen-Activated Protein Kinase (MAPK) pathways are central nodes in the signaling network of eukaryotic cells, because they relay extracellular cues such as growth factors or stresses (Roux and Blenis, 2004; Chen and Thorner, 2007). The metabolic state, the cellular morphology or the cell cycle phase of individual cells can be integrated by the MAPK cascade to finely tune the cellular response (Strickfaden et al., 2007; Nagiec and Dohlman, 2012; Clement et al., 2013). However, the molecular mechanisms that allow these signal integrations are generally unknown.

12 The simplified settings offered by S. cerevisiae provide an ideal platform to study these complex 13 mechanisms. The budding yeast MAPK network is composed of four main pathways active in 14 haploid cells (Saito, 2010). Multiple instances of signal integration have been documented in this 15 model system: the cross-inhibition between two MAPK pathways (Nagiec and Dohlman, 2012), 16 the limitation of signal transmission in low nutrient conditions (Clement et al., 2013; Sharifian et 17 al., 2015) and the tight coupling between cell cycle regulation and MAPK activity (Oehlen and

18 Cross, 1994; Peter and Herskowitz, 1994; Wassmann and Ammerer, 1997; Escoté et al., 2004;

19 Clotet and Posas, 2007; Strickfaden et al., 2007). In this study, we were interested in the

20 interplay between the cell cycle and the mating pathway.

21 Haploid budding yeasts exist in two mating types: MATa and MAT $\alpha$. They produce pheromones

22 (respectively a-factor and $\alpha$-factor) that can be sensed by a mating partner. Activation of a G-

23 protein-coupled receptor by the pheromone leads to the activation of the MAPKs Fus3 and Kss1,

24 via a three-tier kinase cascade recruited to the plasma membrane by the scaffold protein Ste5

25 (Bardwell, 2005; Atay and Skotheim, 2017). The two MAPKs initiate a mating program that

26 includes the transcription of hundreds of genes, the arrest of the cell cycle in G1, the formation

27 of a mating projection and which culminates in the fusion of the two partners.

28 In order to guarantee that each cell that undergoes fusion possesses a single copy of its genome, 29 active Fus3 phosphorylates the Cyclin Kinase Inhibitor (CKI) Far1 which arrests the cells in G1 30 (Peter et al., 1993; Peter and Herskowitz, 1994). In addition, during division, signaling in the 
31 mating pathway is dampened by the action of the Cyclin Dependent Kinase (CDK) Cdc28.

$32 \mathrm{Cdc} 28$, the only CDK in S. cerevisiae, associates with the different cyclins to ensure the proper

33 progression through the cell cycle. Inhibition of the mating pathway is made possible by the

34 association between the CDK and the late-G1 cyclins $\mathrm{Cln} 1$ and $\mathrm{Cln} 2$. The $\mathrm{Cln} 1 / 2-\mathrm{Cdc} 28$

35 complex has been shown to phosphorylate the scaffold protein Ste5, thereby preventing its

36 recruitment to the plasma membrane and thereby preventing the transduction of the signal from

37 the receptor to the MAPK cascade (Strickfaden et al., 2007).

38 Most of the knowledge on the mating-induced cell cycle arrest in G1 and the inhibition of the

39 mating pathway during division has been obtained from population-level measurements, relying

40 on artificial synchronizations of the cell cycle using temperature sensitive mutants, chemical

41 inhibitors or by overexpression of cyclins (McKinney et al., 1993; Peter et al., 1993; Wassmann

42 and Ammerer, 1997; Strickfaden et al., 2007). More recent studies have used single cell

43 measurements to monitor this cross-inhibition, but focused either on Ste5 relocation (Repetto et

44 al., 2018) MAPK activity (Durandau et al., 2015; Conlon et al., 2016) or on CDK activity

45 (Doncic et al., 2015).

46 In this study, we have developed a sensitive assay enabling to quantify in parallel MAPK and

47 CDK activity in non-synchronized live single cells using fluorescent biosensors. By exploiting

48 the natural diversity present in the population, we have been able to cluster cells based on their

49 cell cycle position and monitored their MAPK activity pattern. We could confirm the key role of

50 Far1 for the G1 arrest. However, our data suggest that an additional mechanism working in

51 parallel with the Ste5 phosphorylation is required to limit signaling during S-phase. Furthermore,

52 we highlight the importance of the cross-inhibition between MAPK and CDK for cell-fate

53 decision in the mating process. The interplay between these two activities will determine whether

54 cells induce a mating response or commit to a new cell cycle round.

\section{Results}

\section{Quantifying MAPK activity}

57 In order to quantify mating MAPK activity, we have developed a Synthetic Kinase Activity

58 Relocation Sensor (SKARS). This probe was engineered by combining a specific MAPK

59 docking site, a phosphorylatable Nuclear Localization Signal (NLS) and a Fluorescent Protein 
60 (FP) (Durandau et al., 2015). The docking site consists in the first 33 amino acids from the

61 MAP2K Ste7, which confers specificity towards both Fus3 and Kss1 (Reményi et al., 2005).

62 Under normal growth conditions, the NLS promotes the enrichment of the sensor in the nucleus.

63 Upon activation of the MAPK, specific residues neighboring the NLS are phosphorylated by the

64 targeted MAPK, leading to a decrease in the import rate of the sensor in the nucleus. The

65 presence of the FP allows monitoring nuclear-to-cytoplasmic partitioning of the sensor as

66 function of time (Figure 1A). We use two bright field images and a fluorescence image of the

67 nucleus (Hta2-tdiRFP) to segment the nuclear and cytoplasmic areas in each cell and measure the

68 fluorescence intensity in the other fluorescent images acquired (Pelet et al., 2012). The ratio of

69 mean fluorescence intensities between these two compartments in the RFP channel provides a

70 dynamic measure of MAPK signaling activity in each single cell.

71 One experimental difficulty associated with this sensing strategy is the fact that each cell has a

72 distinct inherent capacity to import the sensor in the nucleus. Therefore, the read-out provided

73 for each cell includes an additional undesired component. In order to correct for this

74 experimental variability, we introduced, in parallel to the functional sensor present in the RFP

75 channel, a non-functional sensor in the CFP channel based on a version of the reporter that

76 cannot bind the kinase (non-docking sensor, Ste $7_{\mathrm{ND}}-\mathrm{SKARS}$, Figure 1B). We define the MAPK

77 activity as the ratio of cytoplasmic-to-nuclear fluorescence of the functional sensor over the ratio

78 of cytoplasmic-to-nuclear fluorescence of the non-docking corrector (Supplementary Figure 1

79 and Methods). This metric provides a relative measure of the combined Fus3 and Kss1 activity

80 independently of the nuclear import capacity of each cell.

81 We have performed time-lapse experiments where cells are stimulated with synthetic pheromone

82 at saturating concentration (1000nM $\alpha$-factor) directly under the microscope. The dynamics of

83 activation of the mating pathway in single cells can be quantified (Figure 1C). In comparison

84 with a signaling dead mutant (ste5s), we observe a clear increase in the median MAPK activity

85 of the population within 5 minutes after the stimulus. However, the few single cell traces plotted

86 in this figure display strikingly different dynamic behaviors, demonstrating well the great

87 heterogeneity in the signaling capacity of individual cells. 
89 Because it has been established for a long time that the cell cycle can influence mating signaling

90 competence, we decided to monitor in the same cells both MAPK and CDK activities to

91 overcome the need for population average measurement and artificial cell cycle synchronization.

92 In order to follow cell cycle progression in an automated and robust manner, we used a

93 fluorescently tagged Whi5, which has been used in many studies as an endogenous relocation

94 probe for the G1 state (Bean et al., 2006; Doncic et al., 2011). Whi5 is enriched in the nucleus of

95 the cells in G1 to repress the expression of specific cell cycle genes. Phosphorylation by the

96 Cln3-CDK complex relieves this repression by promoting the nuclear export of Whi5 (Costanzo

97 et al., 2004; de Bruin et al., 2004) (Figure 1D and E). Whi5 will only shuttle back into the

98 nucleus as cells re-enter in G1. Thus, in addition to the SKARS and its corrector, we tagged

99 Whi5 with mCitrine and measured the cytoplasmic to nuclear fluorescence intensity in the YFP

100 channel as a proxy for CDK activity (Figure 1F). Note that during the division process,

101 fluctuation in CDK activity levels cannot be quantified using this approach, but the Whi5 probe

102 allows us to precisely monitor the exit from G1 and the entry into G1. In the dataset of a two-

103 hour time-lapse movie, we collected single cell traces that displayed a transient nuclear

104 accumulation of Whi5. The rapid accumulation of Whi5 in the nucleus, which corresponds to the

105 sharp decrease in CDK activity occurring at the onset of G1, was used to synchronize

106 computationally the single cell traces (Figure 1F and Supplementary Figure 2). The alignment of

107 the single-cell responses reveals two additional CDK activity drops taking place roughly 90

108 minutes before or after the central trough. This timing matches the expected cell cycle length in

109 these conditions (Charvin et al., 2008). To sum up, this reporter strain allows us to monitor in

110 parallel CDK and mating MAPK activities in single cells, thereby providing an assay to

111 disentangle their interactions.

112 Cell cycle stage clustering

113 In a naturally cycling population of budding yeast cells, all cell cycle stages are represented at a

114 given time point. Therefore, when this population is stimulated with $\alpha$-factor, in a single

115 experiment, we can observe the entire diversity of responses present in the population generated

116 by this extrinsic variability. In a typical time-lapse experiment of 45 minutes, cells are imaged

117 every 3 min and the pheromone is added before the third time point. From such a microscopy

118 dataset, several hundreds of single cells are tracked and pass through a quality control filter. Note

119 that unless stated otherwise, all following experiments with exogenous pheromone stimulation 
120 are performed in a barl $1 \Delta$ background, to maintain a constant extracellular concentration of $\alpha$ -

121 factor in the medium surrounding the cells and were performed in at least three independent

122 experiments.

123 The single cell traces are clustered in silico based on their CDK activity pattern to identify the 124 different cell-cycle stage populations (see Methods). The first cluster consists of cells with low 125 CDK activity throughout the time lapse (G1,20\% of the population). These $G 1$ cells are MAPK 126 signaling competent and display a strong and sustained response of the SKARS upon addition of 127 pheromone (Figure 2A). A second cluster is made of cells with a high CDK activity during the 12845 minutes of the time lapse (Out-of-G1,35\%). These cells display a fast response to the mating 129 pheromone but with a low amplitude. Previous reports have shown, by overexpression of cyclins, 130 that the activity of the CDK can abolish or strongly reduce the activity of the mating pathway 131 (Oehlen and Cross, 1994; Wassmann and Ammerer, 1997). Under physiological conditions, our 132 data show that dividing cells are none-the-less able to activate the MAPK pathway, but only 133 weakly compared to the $G 1$ cells.

134 In addition, our dynamic measurements can reveal interesting signaling patterns by focusing our 135 analysis on cells that transition between these two strong and weak signaling states (G1 and Out136 of-G1, respectively). A third cluster was thus defined with cells that start with a low CDK 137 activity and end with high CDK activity. These cells are thus exiting G1 and enter S-phase at 138 some point during the time lapse (G1-exit 11\%, Figure 2C). Interestingly, these cells respond 139 strongly to the pheromone stimulus, but display a transient MAPK activation behavior. When 140 pheromone is added, the mating pathway is first rapidly activated. However, when the CDK 141 activity builds up in the cell, an inhibition of the MAPK pathway sets in, leading to a return of 142 the MAPK activity to a low level roughly 15 min after the stimulus.

143 The fourth cluster consists in cells that enter in G1 during the time lapse (G1-entry, 31\%). These 144 cells start with high CDK activity and end the time lapse with low CDK activity. This transition 145 can happen at any time point during the experiment. The median MAPK activity in this sub146 population increases gradually with time, while the individual traces display sharp transitions 147 from a low signaling to a high-signaling state (Supplementary Figure 3A). Using the Whi5 148 reporter, the single cell traces were temporally aligned based on the time of G1 entry. As a result, 149 a synchronous increase in MAPK activity is observed when CDK activity stabilizes to low 150 values, corresponding to the G1-phase (Figure 2D and Supplementary Figure 3B). Finally, a fifth 
151 cluster was identified with cells that cycle briefly in G1 during the time lapse (Through-G1, 3\%).

152 No specific behavior in MAPK activity was observed in these cells even when traces are aligned 153 relative to the time of G1 entry (Supplementary Figure 4).

154 Figure 2E allows a direct comparison of the MAPK activity measured with the SKARS in

155 different cell cycle stages. The mean response of the population (thick blue line) is not

156 representative of the behavior of individual cells which can display strikingly different behavior.

157 However, because CDK plays a major role in controlling the signaling output of the mating

158 pathway, clustering based on the CDK activity pattern allows to group together cells that display

159 similar MAPK activity dynamics. Thus, we observe four types of behaviors: strong and sustained

160 activity for $G 1$ cells, weak and sustained activity in Out-of-G1 cells, strong but transient activity

161 in the G1-exit cluster and delayed activation (depending on the timing of CDK activity drop)

162 present in the G1-entry cells.

163

164 In order to compare the results obtained with the SKARS, we performed a similar analysis with

165 two different assays that report on mating pathway activity. Using a fluorescently tagged Kss1

166 (Kss1-mScarlett), the relocation of the MAPK from the nucleus to the cytoplasm upon

167 pheromone stimulus has been monitored in a strain carrying Whi5-mCitrine. This change in

168 cellular compartments of Kss1 has been shown to be a consequence of the disassembly of a 169 complex formed between Dig1/Dig2, Ste12 and Kss1, upon phosphorylation by Fus3 and/or

170 Kss1 (Pelet, 2017) (Supplementary Figure 5 A and B). When comparing the response of the cells

171 in different cell-cycle stages, a similar pattern can be observed between this assay and the

172 SKARS reporter (Supplementary Figure 6). The major difference can be observed in the G1-

173 entry cluster where a gradual increase in signaling activity can be seen upon entry into G1

174 (Supplementary Figure 6D), compared to the sharper dynamics of the SKARS reporter in the

175 same cell cycle stage (Figure 2D). In addition, when comparing the Kss1 relocation of G1 and

176 G1-entry cells relative to the time of pheromone addition (Supplementary Figure 6E), we see that

177 they are almost identical. This suggests that cells late in the division process have fully recovered 178 their signaling ability.

179 The second assay is based on the dPSTR (dynamic Protein Synthesis Translocation Reporter) 180 system which allows to monitor the dynamics of induction of a promoter of interest (Aymoz et 
181 al., 2016). This promoter drives the expression of a small peptide that promotes the relocation of

182 a fluorescent protein in the nucleus of the cell. We use the previously published $\mathrm{p} A G A 1$-dPSTR

183 to monitor the dynamics of mating gene induction. AGA1 has been shown to be strongly induced

184 by $\alpha$-factor in a MAPK dependent manner (Roy et al., 1991; Oehlen et al., 1996; Aymoz et al.,

185 2018). Clustering of the gene expression data based on cell-cycle stage demonstrates a strong

186 expression in G1 and G1-entry clusters, while clusters for Out-of-G1 and G1-exit cells display an

187 attenuated and delayed response (Supplementary Figure 7).

188 The global outcome that can be obtained by comparing these three types of reporters is that full

189 signal competence is observed in the Gl cluster, while Out-of-Gl cells have a reduced ability to

190 signal. In addition, cells exiting G1 will only transiently activate the MAPK. Interestingly, this

191 transient activation is not sufficient to drive gene expression as protein production in the G1-exit

192 cluster is delayed by 20 to 30 minutes.

\section{MAPK activity in the G1-entry cluster}

194 The comparison between the SKARS, the Kss1 relocation and the pAGA1-dPSTR also

195 highlights a discrepancy for the G1-entry cluster. While the two latter assays display a signaling

196 ability that is comparable to the one present in G1 cells for the G1-entry cluster, the SKARS

197 measurements suggest that the MAPK activity is only recovered when CDK activity has

198 dropped, as cells enter in G1.

199 The influence of the cell cycle on the nuclear enrichment of the SKARS could potentially

200 explain this observation. Indeed, a lower enrichment of the corrector can be observed in Out-of-

$201 G 1$ cells compared to G1 cells (Supplementary Figure 8A and B). In the G1-entry cluster, this

202 results in a slow transition from a low to a high nuclear to cytoplasmic ratio (N/C) of the

203 corrector around the time of entry into G1. In signaling dead cells, the exact same behavior is

204 observed for the functional sensor (Supplementary Figure 1B). However, the dynamic of this

205 transition is strikingly different from the dynamic of MAPK activity measured upon G1-entry.

206 While the corrector slowly rises from -10 to $+10 \mathrm{~min}$, the MAPK activity shift takes place

207 between +5 and +10 min after the CDK activity drop. Thus, the fact that these two events are not

208 synchronous, strongly suggests that the sharp increase in MAPK activity is not an artifact from a

209 reduced sensitivity during the division of the cells. 
210 Because, in each individual cell, the sensor and corrector nuclear enrichment are highly

211 correlated (Supplementary Figure 8C), we performed an additional verification and separately

212 analyzed cells with low and high nuclear enrichment of the corrector (Supplementary Figure 8D

213 and E). If the G1-entry behavior was an artifact due to a poor enrichment of the sensor, we would

214 expect that cells with a relatively high nuclear enrichment would not display this behavior or at

215 least to a lower extent. On the contrary, we observe that Gl-entry cells which keep a high N/C of

216 the corrector throughout the time-lapse display a stronger change in MAPK activity 10 minutes

217 after G1 entry.

218 In agreement with the Kss1 and dPSTR assays, previous works (Oehlen and Cross, 1994;

219 Wassmann and Ammerer, 1997; Strickfaden et al., 2007; Conlon et al., 2016), have shown that

220 CDK inhibition of the mating pathway is limited to the S-phase and full signaling competence is

221 recovered in G2-M. The dynamics observed with the SKARS are therefore unexpected. This

222 behavior could be explained by the action of phosphatases acting on the phosphorylated residues

223 present on the SKARS. For instance, the Cdc14 phosphatase leaves the nucleus during mitotic

224 exit to dephosphorylate cyclin-dependent targets (Shou et al., 1999; Visintin et al., 1999; Mohl et

225 al., 2009). If this hypothesis is true, we can envision that other substrates of the MAPK might be

226 the target of phosphatases upon G1-entry and restrict their activity to the G1 phase.

\section{Pheromone dose response}

228 In order to test how the MAPK signaling behavior changes as function of input strength, time-

229 lapse movies were recorded with various concentrations of pheromone. The same cell cycle

230 clustering approach was used for all the dataset. As expected, the amplitude of the MAPK

231 activity decreases with lower pheromone concentrations (Figure 3 A-C). However, other changes

232 can also be observed between the different concentrations. In the G1-phase, a sustained MAPK

233 activity is observed at high doses of pheromone, while the signaling activity declines at lower $\alpha$ -

234 factor doses (Figure 3A). This behavior suggests an interplay between positive and negative

235 feedback loops. Multiple regulatory mechanisms have been identified in the mating pathway

236 (Hao et al., 2003; Bhattacharyya et al., 2006; Yu et al., 2008; Nagiec et al., 2015). None-the-

237 less, it remains difficult to estimate their relative influence on the signaling outcome. Our data

238 suggest that at low $\alpha$-factor concentrations, negative feedbacks are prevalent and contribute to

239 the deactivation of the pathway. At high pheromone concentrations, however, the positive 
240 feedbacks stabilize the system in a high activity state for a long time. Similar experiments were

241 performed in $B A R 1+$ cells, where the presence of the pheromone protease adds another layer of

242 regulation to the system and leads to a faster decline in signaling activity at all concentrations but

243 the highest one, which remains sustained over the course of the time lapse experiment

244 (Supplementary Figure 9).

245 It is well-established that treatment with pheromone prevents cells from entering a new cell cycle 246 round (Hartwell et al., 1974), therefore one expects a difference in the proportion of G1 cells at

247 the end of the experiment between $\alpha$-factor treated and mock treated cells (Figure 3D).

248 Interestingly, we noticed a gradual increase in the fraction of cells retained in G1 as function of

249 pheromone concentration. If we focus on the population of cells starting in G1 (G1 and G1-exit

250 clusters, which roughly represent one third of the population), in the untreated situation $20 \%$ will

251 exit G1, while 9\% remain in G1 for the entire time lapse. Note that these measured percentages

252 are specific to our $45 \mathrm{~min}$ the time lapse. For the sample stimulated with pheromone, this

253 proportion gradually increases to reach $20 \%$ of cells that remain in G1 at $1000 \mathrm{nM}$ versus $10 \%$

254 that can still escape G1 arrest. A similar behavior is observed for cells that are dividing at the

255 onset of the time lapse (Out-of-G1 and G1-entry). The proportion of the cells that enter and stay

256 in G1 (G1-entry) evolves from $12 \%$ to $30 \%$ as pheromone concentration increases. Thus, the

257 stronger MAPK activity measured at high concentrations promotes a larger accumulation of cells

258 in G1 at the end of the time-lapse movie. These experiments clearly illustrate the fact that the

259 level of MAPK activity influences the ability of the cells to initiate division.

260 START as a signal integration point

261 The START event in the cell cycle has been operationally defined as the time when cells become

262 insensitive to a pheromone stimulus and are committed to a new cell cycle round (Hartwell et al.,

263 1974). Multiple cellular events are coordinated around this decision point. Activity of the G1

264 cyclin $\mathrm{Cln} 3$ increases. It triggers the exit of Whi5 out of the nucleus, thereby allowing the

265 transcription of $\mathrm{Cln} 1$ and $\mathrm{Cln} 2$. These two cyclins will in turn drive the transcription of

266 downstream S-phase genes (Dirick et al., 1995; Costanzo et al., 2004; de Bruin et al., 2004). We

267 have shown that the number of cells that are blocked in G1 or commit to a new cell cycle round

268 is dependent on the pheromone concentration and thus on the MAPK activity. In parallel, we

269 observed that the CDK activity, estimated from the Whi5 nuclear enrichment levels, gradually 
270 increases for cells that commit to a new cell cycle round (G1-exit cluster, Figure 4 A-D). At low

271 pheromone concentration, cells can often override the mating signal and enter the cell cycle.

272 However, at high concentrations of $\alpha$-factor only cells that have already reached a sufficient

273 CDK activity will be able to counterbalance the mating signal. In these cells, the CDK promotes

274 the entry in a new division round. Interestingly, at saturating $\alpha$-factor concentrations, some cells

275 display a transient activation of the CDK, suggesting that the addition of the pheromone

276 inhibited the progression of the cell cycle (Figure 4E). A behavior that has been previously

277 characterized by Doncic et al. (Doncic et al., 2011). Taken together these results comfort the idea

278 that START is a signal integration point where the cells balance the relative MAPK and CDK

279 activities to determine their fate: division or mating.

280 Hence, the cross-inhibition between the MAPK and CDK plays a central role in the decision to

281 mate or divide. The molecular mechanisms regulating the interplay between mating and cell

282 cycle have been extensively studied. Phosphorylation and expression of Far1 upon $\alpha$-factor

283 stimulus inhibit the $\mathrm{Cln} 1 / 2-\mathrm{Cdc} 28$ complex to prevent cell cycle progression into S-phase (Chang

284 and Herskowitz, 1990; Tyers and Futcher, 1993; Peter and Herskowitz, 1994). Deletion of FAR1

285 does not affect the MAPK signaling activity of $G 1$ nor of Out-of-G1 cells (Supplementary Figure

286 10A). However, the fraction of cells found in $\mathrm{G} 1$ at the end of the time-lapse experiment is

287 increasing from $25 \%$ to $50 \%$ for WT cells when changing the pheromone concentration to $1 \mu \mathrm{M}$,

288 while it remains below 30\% in far $1 \Delta$ (Supplementary Figure 10B). These results confirm the

289 important role played by Far1 in the G1-arrest during the mating response.

\section{Modulating Cdc28 activity}

291 We have shown above that the efficiency of the cell cycle arrest depends on the level of MAPK

292 activity. We next want to verify how the CDK activity influence the MAPK signal transduction.

293 In budding yeast, a single Cyclin Dependent Kinase, Cdc28, associates with the different cyclins

294 throughout the cell cycle to orchestrate the division of the cell. While Cdc28 is an essential

295 protein, it is none-the-less possible to acutely inhibit its kinase activity by using an analog

296 sensitive allele (cdc28-as (Bishop et al., 2000)). The inhibitor NAPP1 was added to the cells 6

297 minutes after the pheromone stimulus. Upon NAPP1 treatment, Out-of-G1 cells show a rapid

298 relocation of Whi5 in the nucleus, attesting the fact that CDK activity is blocked. In parallel, the 
MAPK activity, that is low during division, increases to a level comparable to the one observed in cells stimulated in G1 (Figure 5A).

301 Additionally, this experiment allows to verify that the transient MAPK activation observed in the G1-exit cluster is shaped by the rising CDK activity. Cells with an increasing CDK activity after

303 pheromone stimulus were clustered. In the DMSO control experiment, this sub-population

304 displays the expected fast activation of the MAPK upon $\alpha$-factor addition followed by a decay to basal activity as CDK activity rises. In the inhibitor treated cells, the MAPK activity decay is blocked upon NAPP1 addition and the MAPK signal rises to reach full activity (Figure 5B). These experiments demonstrate that the activity of $\mathrm{Cdc} 28$ directly and quickly regulates the level of MAPK activity present in the cell.

\section{9}

310

\section{Ste5 inhibition by the $C D K$}

One mechanism of inhibition of the mating pathway by the CDK has been shown to consist in the direct phosphorylation of the scaffold Ste 5 by the Cln1/2-Cdc28 complex (Strickfaden et al., 2007). Eight consensus phosphorylation sites in the vicinity of a plasma membrane binding domain (PM) in Ste5 are targeted by the CDK to alter the charge of this peptide. This phosphorylation prevents the association of Ste5 to the membrane, thereby blocking the signal flow in the pathway. In ste $5 \Delta$ cells, we reintroduced three different alleles of Ste5 at the endogenous locus: the WT copy, the Ste $5_{8 \mathrm{~A}}$ mutant (where all putative phosphorylatable residues were mutated to alanine (Strickfaden et al., 2007)) or a Ste $5_{\mathrm{CND}}$ (where the docking motif of Cln1/2 on Ste 5 has been mutated (Bhaduri and Pryciak, 2011)) (Figure 6A). We monitored the response of the mating pathway for these three alleles with the SKARS, the relocation of Kss1 and the $\mathrm{p} A G A 1$-dPSTR at 10 and $100 \mathrm{nM} \alpha$-factor (Figure 6B, C and D). The wild-type STE5 behaved similarly to the WT parental strains, demonstrating the functionality of the complementation. As expected, signaling activity in $G 1$ cells is minimally influenced by either mutation in Ste 5 because the CDK is inactive. In the G1-exit cluster, the inhibition following the transient activation of the pathway is less pronounced in the two Ste5 mutants than for the wildtype allele. Generally, the behavior is more pronounced at $10 \mathrm{nM}$ than at $100 \mathrm{nM} \alpha$-factor and the Ste $5_{8 \mathrm{~A}}$ mutant displays a weaker inhibition by the CDK than the Ste $5_{\mathrm{CND}}$ mutant.

A recent report has demonstrated that the combined action of Fus 3 and $\mathrm{Cdc} 28$ is required to phosphorylate the eight residues Ste5 in the vicinity of the PM domain (Repetto et al., 2018). 
329 However, our data suggest that an additional mechanism might contribute to limit the signal

330 transduction of the mating pathway in the early stage of the division process. This phenomenon

331 is best observed at low concentrations of pheromone where the activity of the MAPK cascade is

332 weaker. This behavior has not been detected previously, probably because most experiments

333 have been performed at saturating levels of pheromone. However, in mating mixture where

334 pheromone concentration is low, this mechanism could contribute to the cell fate decision. In

335 order to identify other potential targets of the CDK, we have tested various alleles of Ste20

336 because it has often been suggested as a potential target for this regulation (Oehlen and Cross,

337 1998; Wu et al., 1998; Oda et al., 1999) (Supplementary Figure 11A and C) and we have also

338 tested the influence of phosphorylation sites on Ste7 without measuring any detectable changes

339 in signaling activity between mutants (Supplementary Figure 11B and D).

340 To summarize, START is the integration point where MAPK and CDK activities are compared

341 to engage in a specific cellular fate. Far1 and Cdc28 are key players in this cross-inhibition of the

342 two pathways. farl $1 \Delta$ cells cannot arrest their cell cycle at START. None-the less, a number of

343 Far1-independent mechanisms for cell cycle arrest have been documented (Tyers, 1996; Oehlen

344 et al., 1998; Cherkasova et al., 1999). Cdc28 is solely responsible for the inhibition of the mating

345 pathway by the cell cycle and, up to now, only a single target, Ste5, has been convincingly

346 shown to regulate this process. Our data indicate that other mechanisms, that remain to be

347 identified, contribute to the repression of MAPK activity in dividing cells.

\section{MAPK activity in mating}

349 After studying the response of cells stimulated by exogenous pheromone, we next wanted to

350 understand how the cell cycle and the mating pathway were regulated during mating and how

351 this cross-inhibition allowed an efficient cell-fate selection in these physiological conditions. In

352 order to achieve this, we have imaged the MATa cells bearing the SKARS, the corrector and the

353 Whi5 marker in presence of a MAT $\alpha$ partner expressing constitutively a cytosolic CFP at high

354 levels. In Figure 7A, thumbnails of such an experiment are displayed. The fusion events (arrow

355 heads) can be detected by observing a sudden increase in CFP signal in the MATa cells. In the

356 frame preceding the two fusion events displayed in Figure 7A, we observe that the MATa cells of

357 interest are in G1 (nuclear Whi5) and display a high MAPK activity (nuclear depletion of the

358 SKARS). 
359 The dynamic measurements of the mating process allowed us to monitor how cells reach this

360 state. Using our automated image analysis pipeline, we have detected more than one hundred

361 fusion events and curated them manually to remove any artifacts due to a mis-segmentation of

362 the cells. The single cell traces of these events have been computationally aligned relative to the

363 fusion time. Time zero corresponds to the last frame before the increase in CFP intensity,

364 because it is the last time point where MAPK activity can be reliably quantified (Figure 7B). As

365 a reference, the median MAPK activity of cells imaged under the same conditions but in absence

366 of a mating partner is plotted. In the fusing cell, a gradual increase in MAPK activity starts 40 to

36760 minutes prior to fusion. In parallel, we observe that the median CDK activity is low for all

368 time-points. However, the 75 percentile stabilizes to a low value 40 minutes prior to fusion,

369 denoting that a fraction of the population enters in G1 within the hour preceding the fusion.

370 From the analysis of these fusion events, it becomes clear that the enrichment in G1 state prior to

371 fusion occurs in cells that experience a low level of MAPK activity, or, in other words, when

372 cells are surrounded by a low concentration of mating pheromone. In the exogenous stimulation

373 experiments, we have shown that at low pheromone concentrations, a fraction of the cells do not

374 commit to the mating response and keep proliferating. We verified if in the mating mixtures non-

375 fusing cells were influenced by the presence of the mating partners. To achieve this, we

376 monitored the CDK activity in cells that were cycling through G1. Interestingly, we observe that

377 the G1 state of these cells is prolonged and displays a great variability (Figure 7C). In these

378 cycling cells, the CDK activity remains lower in cells in mating mixtures compared to the same

379 cells imaged alone (Figure 7D). In parallel, a weak activation of the mating pathway can be



381 display a fast cycling through G1 compared to the WT cells because they remain insensitive to

382 the presence of the partners. In this background, the deletion of the scaffold Ste5 leads to an

383 absence of activity of the MAPK Fus3 and Kss1 which cannot counteract the rise of the CDK

384 activity at START.

\section{Discussion}

386 In this study, we performed dynamic single cell measurements with live-cell imaging. These

387 time lapse movies were automatically quantified, allowing the clustering and in silico

388 synchronization of hundreds of single cell traces. The ability to follow the response of individual 
cells in a population of naturally cycling cells has enabled us to monitor the influence of the cell cycle on the mating process with minimal perturbations. Importantly, the correlation of multiple signaling activities within the same cell by combining fluorescent reporters for CDK and MAPK activities allowed us to identify different MAPK signaling patterns, which demonstrates the ability of the MAPK cascade to integrate the CDK activity to deliver the required signaling output. The molecular mechanisms of some of these integrations have been identified previously, but our quantitative measurements suggest that additional mechanisms contribute to the interplay between MAPK and CDK.

We have verified with exogenous stimulation experiments performed with various concentrations of pheromone that START is a central integration point where cells compare the relative levels of MAPK and CDK activities to decide on their cellular fate: proliferation or mating. It remains to be precisely determined which molecular mechanisms control this decision.

401 On the MAPK side, our data agree with the numerous previous studies that demonstrated that the MAPK activity controls the CDK via the protein Far1 (Peter et al., 1993; Peter and Herskowitz,

403 1994). The mating pathway regulates both the level of Farl and its phosphorylation status. What 404 remains to be understood is how each one of these changes influence the decision made by the 405 cell.

406 On the CDK side, Cdc28 kinase activity is directly regulating the signal flow in the MAPK 407 pathway. Blocking Cdc28 activity relieves this inhibition allowing recovering full MAPK 408 activity, within minutes after addition of the chemical inhibitor (Figure 5). This suggests a very 409 direct mechanism of action on the mating pathway. The primary candidate for this process has 410 been Ste5. However, our quantitative measurements speak for an additional mechanism 411 detectable mostly at low pheromone concentrations. Other potential candidate targets of Cdc28 412 could be the G-protein and the receptor, or other proteins in the MAPK signal transduction 413 cascades.

414 The experiments performed with mating mixtures also illustrate that the interplay between the 415 CDK and the MAPK are central elements in the cell fate selected by the cells. Our data indicate 416 that the commitment to the mating program is taking place at low levels of MAPK signaling 417 activity, where we have shown with exogenous pheromone stimulation, that the CDK can 418 frequently override the MAPK arrest. The sensing of the pheromone secreted by nearby mating 419 partners triggers a low level of MAPK activity, including the activation of positive and negative 
420 feedback regulation mechanisms (Figure 8). In the cells that will successfully mate, we observe 421 that the MAPK activity is progressively rising and thereby preventing an activation of the CDK 422 that would promote an entry in the cell cycle. The polarized secretion of pheromones by both 423 partners contributes further to this increase in MAPK activity that reaches its maximum shortly

424 before the fusion of the two cells. Cells which are at some distance from a potential partner 425 experience lower levels of pheromone. Internal feedback loops together with the secretion of the

426 Bar1 protease contribute to dampen the MAPK activity thus when cells reach the cell-cycle commitment point the CDK can override this low mating signaling activity and promote a new cell cycle round.

429 Throughout all eukaryotes, MAPK signaling and the cell cycle are highly conserved cellular 430 processes. The homolog of Fus3 in mammalian cells is ERK. ERK is probably best known for its 431 implication in cell growth and division in somatic cells (Meloche and Pouysségur, 2007) and 432 hyperactivating mutation in the ERK pathway are found in numerous cancers (Davies et al., 433 2002). However, the ERK pathway can also inhibit cell cycle progression. One well-known 434 example is the stimulation of PC-12 cells with NGF, which results in a prolonged activation of 435 ERK, promoting a cell cycle arrest and differentiation into neuronal cells (Marshall, 1995; 436 Pumiglia and Decker, 1997). More generally, during development MAPK pathways play a 437 central role in the commitment of cells into specific lineage and this is often performed in tight correlation with the cell cycle (Orford and Scadden, 2008). Correlating dynamic measurements of CDK and MAPK activities in these cells could reveal the underlying mechanisms that allow 440 the MAPK to integrate cell cycle cues to modulate the signaling outcome.

\section{Materials and Methods}

\section{$442 \quad$ Strains and plasmids}

443 Yeast strains and plasmids are listed in Supplementary Tables 1 and 2. SKARS plasmids were

444 constructed by cloning the different sensors from Durandau et al (Durandau et al., 2015) into a 445 pSIV vector backbone (Wosika et al., 2016). The sensor and the corrector were assembled by 446 cloning the $S T E 7_{1-33}-N L S-N L S-m C h e r r y$ or the $S T E 7_{N D}-N L S-N L S-C F P$ (HindIII-KpnI) 447 sequences downstream the pRPS6B promoter into the pSIV-URA (SacI-KpnI). The plasmid 448 pED141 containing both sensor and corrector was obtained by cloning the pRPS6B -STE7 ${ }_{N D}-$ 
NLS-NLS-CFP into the second MCS of pED92 (AatII-SphI). Plasmids were transformed in yeast of W303 background expressing the Hta2-iRFP (yED136) or the Hta2-tdiRFP (yED152). Whi5

451 was tagged with mCitrine using pGTT-mCitrine plasmid (Wosika et al., 2016). Kss1 was tagged

452 with mScarlet (pGTL-mScarlet). pAGA1 induction was monitored by integrating the $\mathrm{p} A G A 1$ -

$453 \mathrm{dPSTR}^{\mathrm{R}}$ in the URA3 locus (Aymoz et al., 2018).

454 Genomic deletions were constructed in cells bearing the sensors using KAN or NAT resistance 455 cassettes (Longtine et al., 1998; Goldstein and McCusker, 1999). Plasmids containing the coding

456 sequence of Ste5, Ste20 and Ste7 were obtained by amplification of a chromosome fragment

457 spanning from -100bp (into the promoter) to the end of the ORF and cloned using PacI-NheI

458 sites into the pGTH-CFP (replacing the fluorescent protein). Mutated variants were then obtained

459 by replacing a portion of the plasmid DNA coding sequence by a synthetic double-stranded DNA

460 fragment bearing the desired mutations. Plasmids were integrated into the genome of yeast by

461 replacing the NAT or KAN cassettes used to delete the native gene using homology regions in

462 the promoter of the gene and in the TEF terminator of the deletion cassette, which is also present

463 on the pGTH vector.

\section{Sample preparation}

465 The cells were grown to saturation in overnight cultures at $30^{\circ} \mathrm{C}$ in synthetic medium (YNB:

466 CYN3801, CSM: DCS0031, ForMedium). They were diluted in the morning to $\mathrm{OD}_{600}=0.05$ and

467 grown for at least four hours before starting the experiments. For experiments in wells, 96-well

468 plates (MGB096-1-2LG, Matrical Bioscience) were coated with a filtered solution of

469 Concanavalin A $(0.5 \mathrm{mg} / \mathrm{ml}, 17-0450-01$, GE Healthcare $)$ for 30min, rinsed with $\mathrm{H}_{2} \mathrm{O}$, and dried

470 for at least 2 hours. Before the experiment, the cultures were diluted to an $\mathrm{OD}_{600}$ of 0.05 , and

471 briefly sonicated. A volume of $200 \mu 1$ of culture was loaded into each well. Cells were left

472 settling 30-45 minutes before imaging. To stimulate the cells, $100 \mu 1$ of inducing solution was

473 added to the wells. For $\alpha$-factor stimulation, final concentration is indicated into the figure

474 legend. To inhibit the $c d c 28-a s$, a 25mM stock solution of NAPP1 (A603004, Toronto Research

475 Chemical) in DMSO was diluted in SD-medium and added to the wells. The final working

476 concentration is indicated in the figure legend. For control experiments, cells were treated with

477 DMSO $0.16 \%$ in SD-full. 
478 For mating assay experiments, $\log$ phase cultures of MATa and MAT $\boldsymbol{\alpha}$ (ySP694) were diluted to

479 OD 0.1 in $500 \mu 1$ SD-full. Cells were spun down for $2 \mathrm{~min}$ at $3000 \mathrm{rpm}$. The supernatant was

480 removed from the cultures and MAT $\alpha$ cells were resuspended in $10 \mu 1$ of SD-full. These $10 \mu 1$

481 were then used to resuspend the MATa cells. $1 \mu 1$ of this mating mixture was placed on an agar

482 pad. The pad was then placed upside down into the well of a 96-well plate. To prepare the pad, a

$4832 \%$ agarose mixture in SD-full was heated for $5 \mathrm{~min}$ at $95^{\circ} .150 \mu 1$ liquid was placed in a home-

484 made aluminum frame. After cooling down, the $7 \mathrm{~mm}$ square pad was gently extracted from the

485 frame. Typically, 6 to 8 mating pads were prepared and imaged in parallel.

Microscopy

487 Images were acquired with a fully automated inverted epi-fluorescence microscope (Ti-Eclipse,

488 Nikon), controlled by Micromanager software (Edelstein et al., 2010) and placed in an

489 incubation chamber set at $30^{\circ} \mathrm{C}$, with a $40 \mathrm{X}$ oil objective and appropriate excitation and

490 emission filters. The excitation was provided by a solid-state light source (SpectraX, Lumencor).

491 The images were recorded with a sCMOS camera (Flash4.0, Hamamatsu). A motorized XY-

492 stage allowed recording multiple fields at every time points. Cy5p5 (200ms), CFP (100ms), RFP

493 (100ms), YFP (300ms) and two brightfield (10ms) images were recorded at time intervals of 3 or

4945 minutes.

\section{Data analysis}

496 Time-lapse movies were analyzed with the YeastQuant platform (Pelet et al., 2012). The cell

497 nuclei were segmented by thresholding from Cy5p5 images. The contour of the cell around each

498 identified nucleus was detected using both brightfield images. The cytoplasm object was

499 obtained by removing the nucleus object expanded by two pixels from the cell object. The nuclei

500 were tracked across all the frames of the movie. Multiple features of each object were quantified.

501 Note that for mating pad experiments, the MAT $\alpha$ cells were not segmented as they do not express

502 the Hta2-iRFP. Dedicated scripts were written in Matlab R2016b (The Mathworks) to further

503 analyze the data. Except for Figure 7, only cells tracked from the beginning to the end of the

504 movie were taken into consideration. In addition, a quality control was applied on each trace and

505 only the traces with low variability in nuclear and cell area, and Cy5p5 nuclear fluorescence 
506 were kept for further analysis. On average, $65 \%$ of the cells tracked from the beginning to the

507 end of the time lapse passed the quality control.

508 For each cell, the average nuclear intensity in the fluorescent channel corresponding to the

509 SKARS, the Corrector and Whi5-mCitrine were divided by the average intensity in the

510 cytoplasm for every time point (ratio N/C). To quantify the MAPK activity, the Adjusted ratio

511 for each individual cell was obtained by dividing the ratio N/C of the Corrector by the ratio N/C

512 of the SKARS. The CDK activity of each cell was defined as the inverse of the Whi5-mCitrine

$513 \mathrm{~N} / \mathrm{C}$ ratio. The initial CDK activity was defined as the average of three time-points before the

514 stimulus (3-6 minutes before $\alpha$-factor stimulus). All these quantities are unitless numbers, since

515 they are ratios between fluorescence intensities obtained from the microscope camera. We used

516 the symbol [-] to represent this lack of units.

\section{CDK activity-based clustering and synchronization}

518 When plotting a histogram using the Whi5 ratio N/C (Nuclear intensity over Cytoplamsic

519 intensity) of all cells at all time points, we identified two populations. To properly distinguish

520 between ratios corresponding to nuclear Whi5 versus ratios corresponding to cytoplasmic Whi5,

521 we first separated ratio values using a fixed threshold (threshold=2). However, this value

522 overestimated the limit of what could be considered as nuclear Whi5. We then identified cells

523 with ratios below this threshold at all time points and replotted a histogram of all Whi5 ratio N/C

524 values from this sub-population. This method enables to enrich the sample with low N/C ratio.

525 This second histogram presented a clearer overview of the values corresponding to cytoplasmic

526 Whi5. We then calculated the derivative of the histogram and identified the position of fifth-

527 lowest derivative value. The corresponding Whi5 ratio N/C was used as a final threshold to

528 separate nuclear to cytoplasmic Whi5 values. This procedure enables to correct for slight

529 differences between experiments mostly due to the chosen experimental settings (96 well-plate

530 or pad experiments). Globally, the procedure adjusted the threshold between 2 and 1.7. If the

531 Whi5 ratio N/C was above the threshold, corresponding to a low CDK activity value $(\mathrm{C} / \mathrm{N})$ and

532 thus the cell was considered in G1. On the opposite, values below the threshold indicate that the

533 cell was Out-of-G1. G1 cells sub-population was defined by single cell traces which remained

534 above the threshold for the entire duration of the time lapse. Conversely, Out-of-G1 cells cluster

535 was defined by traces that stay below this threshold. The other single cell traces are scanned for 
536 G1-entry and G1-exit events. If a pattern of two values below the threshold followed by two

537 values above the threshold was found, we considered that the cell belonged to the G1-entry

538 cluster. The same strategy was used to identify the G1-exit sub-population, using a pattern of two

539 values above the threshold followed by two values below the threshold. Cells in the G1-entry

540 cluster were defined as having a single event of G1-entry. Cells in the G1-exit cluster were

541 selected as having only one event of G1-exit. Finally, the Through-G1 cluster is made of cells

542 that display a G1-entry event followed by a G1-exit event. Because the duration of the time-lapse

543 experiments is much shorter than the cell cycle of the yeast (45 min vs. $90 \mathrm{~min}$ ), we considered

544 that the number of events cannot exceed two. Cells which do not follow this criterion were

545 rejected from the analysis. Depending on the experiment, we rejected a maximum of $5 \%$ of the

546 total population. The position of the G1-entry was used to create new time vectors for

547 synchronization of the G1-entry and the Through-G1 single cell traces. We extracted the

548 individual time vector for each cell and subtracted the time at which the G1-entry event occurred.

\section{Detection of fusion events in mating assays}

550 MAT $\alpha$ cells express the CFP fluorescent protein under the control of a strong promoter. When

551 MATa and MAT $\alpha$ cells fuse the CFP from the MAT $\alpha$ cell diffuses into the partner leading to a

552 rise of the fluorescence into the segmented MATa. Fusing MATa cells were first detected

553 bioinformatically by identifying a sudden increase of the CFP intensity in the nucleus object. We

554 chose the nucleus object rather than the whole cell to avoid that mis-segmentation triggers a

555 misleading increase in CFP intensity not linked to a fusion event. Fusion event time was defined

556 as the time point at which the difference in the CFP nuclear signal between two consecutive time

557 points exceed 200, while remaining below 700. Traces of fusing cells were selected as they

558 contain a single detected fusion event. We filtered out cells with aberrant absolute and derivative

559 nuclear CFP fluorescence signal or tracked for less than 15 min prior or after the fusion time.

560 CFP images corresponding to selected cells were checked manually to reject false positives that

561 could not be detected during the bioinformatics analysis. The position of the fusion event into the

562 time vectors of individual fusing cells was used as a time reference to align temporally the

563 MAPK activity and CDK (Figure 7).

\section{Acknowledgments}


565 We thank all members of the Pelet and Martin labs for helpful discussions and comments on the manuscripts, Marta Schmitt and Clémence Varidel for technical assistance. This study was supported by Swiss National Science Foundation grants (PP00P3_139121) and the University of

568 Lausanne.

\section{Author contributions}

570 ED and SP conceived and study and wrote the manuscript. ED constructed the strains and performed the experiments with exogenous stimulation of pheromone. SP performed the mating

572 assay experiments. ED analyzed the single cell data.

573 The authors have declared that no competing interests exist.

\section{$574 \quad$ Figure Legends}

\section{Figure 1: Dynamic MAPK and CDK activity measurements in live single cells.}

576 A. Schematic representation of the Synthetic Kinase Activity Relocation Sensor (SKARS).

577 B. Epi-fluorescence microscopy images of WT cells expressing a Hta2-tdiRFP (nucleus), a

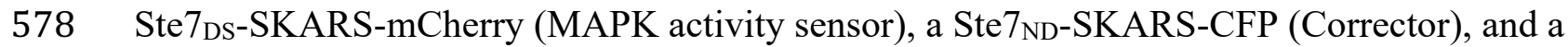

579 Whi5-mCitrine (CDK activity). Log-phase cells were placed in a microscopy well-plate. During

580 the time lapse, cells were stimulated with $1 \mu \mathrm{M} \alpha$-factor (time 0 ).

581 C. MAPK activity was quantified from time-lapse movies acquired with a WT strain (red, Nc=

582 191) and a mating signaling dead mutant (ste5 $\Delta$, blue, $\mathrm{Nc}=437)$ (See Methods and

583 Supplementary Figure 1). For all similar graphs, the solid line represents the median of the

584 population, the shaded area delimits the 25 and 75 percentiles of the population. Dashed lines

585 display the response of a few single cells. Nc represents the number of single-cell traces

586 analyzed.

587 D. Schematic representation of the CDK activity sensor (Whi5-mCitrine).

588 E. Microscopy images of the same strain presented in B. The thumbnails are extracted from a

$589120 \mathrm{~min}$ time-lapse movie of unstimulated cells dividing under normal growth conditions.

590 F. CDK activity reported as function of time relative to G1. Single cell traces that exhibit at least

591 one trough of CDK activity were selected $(\mathrm{Nc}=70)$. The traces were synchronized relative to the

592 time at which the CDK activity reaches its minimum value (see Supplementary Figure 2). 
594 Figure 2: Dynamics of MAPK activity monitored by the SKARS in different cell cycle 595 phases.

596 A-D. MAPK activity was quantified for cells in an asynchronously growing culture stimulated

597 with $1 \mu \mathrm{M} \alpha$-factor at time 0.738 single cell traces were clustered by comparing the CDK 598 activity prior and after stimulation (see Methods). $G 1$ cells (A, Nc $=143$ ) retain a low CDK 599 activity throughout the time lapse, while Out-of-G1 cells $(\mathrm{B}, \mathrm{Nc}=256)$ maintain a high CDK 600 activity. G1-exit cells $(\mathrm{C}, \mathrm{Nc}=76)$ start with low and finish with high $\mathrm{CDK}$ activity. Conversely, 601 Gl-entry cells (D, Nc =249) start with high and finish with low CDK activity. In this sub602 population, the traces are aligned relative to the time of G1-entry. The median (solid line) MAPK 603 activity (red) and CDK activity (green) and the 25-75 percentiles (shaded area) of each sub604 population are plotted.

605 E. Summary of the various dynamic MAPK activities observed upon addition of $1 \mu \mathrm{M} \alpha$-factor 606 at time 0 in the four main cell cycle clusters. Each line corresponds to the mean of the medians of 6075 biological replicates. Error bars represent the standard deviations of the medians. The 608 percentages in the legend indicate the relative proportion of each cluster in the population.

609 Figure 3: Commitment to cell cycle is pheromone dose-dependent.

610 A-C. Pheromone dose-dependent dynamic MAPK activity in different phases of the cell cycle.

611 Experiments and clustering analysis were performed as in Figure 2. Different $\alpha$-factor

612 concentrations were used to stimulate cells. Median MAPK activity from at least five replicates

613 were averaged for each curve. The error bars represent the standard deviations between the 614 replicates.

615 D. Fractions of cells in each sub-population as function of the pheromone concentration for our $61645 \mathrm{~min}$ time lapse. The prevalence of each cluster is calculated from the means of at least three 617 replicates. 
620 A-C. Dynamic CDK activity of cells exiting G1 at various pheromone concentrations. Single cell

621 traces (dashed lines) and the median of the sub-population (solid line) are presented in each

622 graph. Note the decreased variability in CDK activity patterns as pheromone concentration

623 increases.

624 D. Initial CDK activity (mean of the three points before stimulus) measured in the G1-exit cluster

625 for different $\alpha$-factor concentrations. The distribution of the initial CDK of G1-exit cells for one

626 replicate for each concentration is presented as a boxplot. The dots correspond to the median

627 initial CDK activity of additional replicates.

628 E. CDK activity of cells attempting to exit G1. The dynamic CDK activity of G1 cells from

629 multiple 1000nM $\alpha$-factor experiments were pooled. The Attempt G1-exit sub-population (red)

630 contains cells with an initial CDK activity above 0.35. Four single cell traces from the Attempt

631 G1-exit sub-population (dashed lines) that display a transient peak in CDK activity around the

632 time of pheromone addition are plotted. The median CDK activity for the G1 (blue) and G1-exit

633 (green) clusters from Figure 2 are plotted for comparison.

635 Figure 5: Regulation of MAPK signal transduction by the CDK.

636 A-B. Dynamic CDK and MAPK activities after $c d c 28$-as chemical inhibition. Cells expressing 637 an analog sensitive allele of $\mathrm{Cdc} 28(\mathrm{cdc} 28$-as) are imaged every two minutes for 50 minutes.

638 Cells are stimulated with 100nM $\alpha$-factor at time 0. Six minutes later either NAPP1 (10 $\mu \mathrm{M}$, 639 solid line and shaded area) or DMSO $(0.04 \% \mathrm{v} / \mathrm{v}$, dashed line $)$ is added. MAPK and CDK 640 activities of cells starting Out-of-G1 (mean $\mathrm{CDK}_{\mathrm{t}<0}>0.5$, A, NAPP1: Nc = 448, DMSO: Nc =

641 381), or exiting G1 upon pheromone addition (mean $\mathrm{CDK}_{\mathrm{t}<0}<0.5$ and mean $\mathrm{CDK}_{0<\mathrm{t}<6}>0.5$, B,

642 NAPPI: $\mathrm{Nc}=39$, DMSO: $\mathrm{Nc}=16$ ) are plotted.

643 Figure 6: Dynamic MAPK activity of cells expressing non-phosphorylatable Ste5 alleles.

644 A. Schematic representation of the wild-type Ste5 scaffold protein structure compared to the 645 Ste $5_{8 \mathrm{~A}}$ mutant with 8 non-phosphorylatable residues in the vicinity of the plasma membrane 646 domain and the Ste 5 CND variant with point mutations in the $C \ln 1 / 2$ docking motif. 
647 B-D. Dynamics of mating pathway activity in G1 (solid line) and G1-exit cells (dashed line)

648 measured by the SKARS (B), Kss1 relocation (cytoplasmic over nuclear intensity, C) and

$649 \mathrm{p} A G A 1-\mathrm{dPSTR}^{\mathrm{R}}$ nuclear accumulation (nucleus minus cytoplasmic intensity, D). Cells

650 expressing either the Ste $5_{\mathrm{WT}}$ (blue), Ste $5_{8 \mathrm{~A}}$ (red), or Ste $5_{\mathrm{CND}}$ allele (yellow) were treated with

651 10nM (left) and 100nM (right) pheromone. Cell cycle clustering was performed as in Figure 2.

652 Median MAPK activity from at least three replicates were averaged for each curve. The error

653 bars represent the standard deviations between the replicates.

\section{Figure 7: CDK and MAPK cross-regulation in mating conditions}

655 A. Microscopy time-lapse images of two fusion events between MATa cells expressing the 656 various sensors mixed with MAT $\alpha$ strain expressing a cytoplasmic CFP. The fusion can be 657 detected when the CFP from the MAT $\alpha$ diffuses in the MATa partner (White arrows). The 658 indicated time is relative to the last time point preceding the second fusion event.

659 B. Dynamic MAPK and CDK activities in MATa cells undergoing fusion detected by a sharp 660 increase in CFP intensity (blue) (See Method). MAPK (red) and CDK (green) activities of the

661 fusing cells were synchronized relative to the last time point before the fusion occurs $(\mathrm{Nc}=127)$.

662 The dashed orange line and the shaded area around it correspond to the median and 25-75

663 percentiles (averaged across multiple time points) of the MAPK activity in MATa cells in

664 absence of mating partners, but imaged in the same conditions.

665 C-D. Duration of the G1 phase of non-fusing cells. Non-fusing cells transiting through G1 were 666 identified as previously (Figure 3C and Methods). C. The time separating the G1 entry and G1 667 exit is calculated from each single cell trace ( $\Delta t_{\text {exit }}$ - entry $)$ and averaged. The means $\Delta t_{\text {exit }}$ - entry 668 from at least three replicates are plotted as boxplot. D. The dynamic CDK activity from single 669 cells are synchronized relative to the G1 entry. Error bars are standard deviation of at least three 670 medians replicates.

671 E. MAPK activity in non-fusing MATa cells transiting through G1. Single cell traces are 672 synchronized relative to the G1 entry and averaged as in Figure 2D. Error bars are standard 673 deviation of at least three medians replicates.

674 In these mating experiments, all strains are BAR1. 


\section{Figure 8: Scheme of the interplay between CDK and MAPK during mating}

676 MATa cells respond to a pheromone gradient secreted by a MAT $\alpha$ mating partner. The strength

677 of the MAPK activity will depend on the level of $\alpha$-factor sensed by the cells and the

678 contribution of positive and negative feedback. At START, relative CDK and MAPK activities

679 will be compared in order for the cell to select a fate: division or mating.

\section{References}

681 Atay, O., and Skotheim, J. M. (2017). Spatial and temporal signal processing and decision making by MAPK 682 pathways. J Cell Biol 216, 317-330.

683 Aymoz, D., Solé, C., Pierre, J.-J., Schmitt, M., de Nadal, E., Posas, F., and Pelet, S. (2018). Timing of gene

684 expression in a cell-fate decision system. Molecular Systems Biology 14, e8024.

685 Aymoz, D., Wosika, V., Durandau, E., and Pelet, S. (2016). Real-time quantification of protein expression at the

686 single-cell level via dynamic protein synthesis translocation reporters. Nature Communications 7, 11304.

687 Bardwell, L. (2005). A walk-through of the yeast mating pheromone response pathway. Peptides 26, 339-350.

688 Bean, J. M., Siggia, E. D., and Cross, F. R. (2006). Coherence and timing of cell cycle start examined at single-cell 689 resolution. Mol Cell 21, 3-14.

690 Bhaduri, S., and Pryciak, P. M. (2011). Cyclin-specific docking motifs promote phosphorylation of yeast signaling 691 proteins by G1/S Cdk complexes. Curr Biol 21, 1615-1623.

692 Bhattacharyya, R. P., Reményi, A., Good, M. C., Bashor, C. J., Falick, A. M., and Lim, W. A. (2006). The Ste5

693 scaffold allosterically modulates signaling output of the yeast mating pathway. Science $311,822-826$.

694 Bishop, A. C. et al. (2000). A chemical switch for inhibitor-sensitive alleles of any protein kinase. Nature 407, 395-

695401.

696 Chang, F., and Herskowitz, I. (1990). Identification of a gene necessary for cell cycle arrest by a negative growth

697 factor of yeast: FAR1 is an inhibitor of a G1 cyclin, CLN2. Cell 63, 999-1011.

698 Charvin, G., Cross, F. R., and Siggia, E. D. (2008). A microfluidic device for temporally controlled gene expression 699 and long-term fluorescent imaging in unperturbed dividing yeast cells. PLoS ONE 3, e1468.

700 Chen, R. E., and Thorner, J. (2007). Function and regulation in MAPK signaling pathways: lessons learned from the 701 yeast Saccharomyces cerevisiae. Biochim Biophys Acta 1773, 1311-1340.

702 Cherkasova, V., Lyons, D. M., and Elion, E. A. (1999). Fus3p and Kss1p control G1 arrest in Saccharomyces

703 cerevisiae through a balance of distinct arrest and proliferative functions that operate in parallel with Farlp.

704 Genetics 151, 989-1004.

705 Clement, S. T., Dixit, G., and Dohlman, H. G. (2013). Regulation of yeast G protein signaling by the kinases that

706 activate the AMPK homolog Snf1. Science Signaling 6, ra78.

707 Clotet, J., and Posas, F. (2007). Control of cell cycle in response to osmostress: lessons from yeast. Meth

708 Enzymol 428, 63-76. 
Conlon, P., Gelin-Licht, R., Ganesan, A., Zhang, J., and Levchenko, A. (2016). Single-cell dynamics and variability of MAPK activity in a yeast differentiation pathway. Proc Natl Acad Sci USA 113, E5896-E5905.

Costanzo, M., Nishikawa, J. L., Tang, X., Millman, J. S., Schub, O., Breitkreuz, K., Dewar, D., Rupes, I., Andrews, B., and Tyers, M. (2004). CDK activity antagonizes Whi5, an inhibitor of G1/S transcription in yeast. Cell 117, 899-913.

714 Davies, H. et al. (2002). Mutations of the BRAF gene in human cancer. Nature 417, 949-954.

715 de Bruin, R. A. M., McDonald, W. H., Kalashnikova, T. I., Yates, J., and Wittenberg, C. (2004). Cln3 activates G1716 specific transcription via phosphorylation of the SBF bound repressor Whi5. Cell 117, 887-898.

717 Dirick, L., Böhm, T., and Nasmyth, K. (1995). Roles and regulation of Cln-Cdc28 kinases at the start of the cell

718 cycle of Saccharomyces cerevisiae. Embo J 14, 4803-4813.

719 Doncic, A., Atay, O., Valk, E., Grande, A., Bush, A., Vasen, G., Colman-Lerner, A., Loog, M., and Skotheim, J. M. (2015). Compartmentalization of a bistable switch enables memory to cross a feedback-driven transition. Cell 160, $1182-1195$.

722 Doncic, A., Falleur-Fettig, M., and Skotheim, J. M. (2011). Distinct interactions select and maintain a specific cell 723 fate. Mol Cell 43, 528-539.

Durandau, E., Aymoz, D., and Pelet, S. (2015). Dynamic single cell measurements of kinase activity by synthetic 725 kinase activity relocation sensors. BMC Biol. 13, 55.

726 Edelstein, A., Amodaj, N., Hoover, K., Vale, R., and Stuurman, N. (2010). Computer control of microscopes using $727 \mu$ Manager. Curr Protoc Mol Biol Chapter 14, Unit14.20.

Escoté, X., Zapater, M., Clotet, J., and Posas, F. (2004). Hog1 mediates cell-cycle arrest in G1 phase by the dual

729 targeting of Sic1. 6, 997-1002.

730 Goldstein, A. L., and McCusker, J. H. (1999). Three new dominant drug resistance cassettes for gene disruption in 731 Saccharomyces cerevisiae. Yeast 15, 1541-1553.

732 Hao, N., Yildirim, N., Wang, Y., Elston, T. C., and Dohlman, H. G. (2003). Regulators of G protein signaling and 733 transient activation of signaling: experimental and computational analysis reveals negative and positive feedback 734 controls on G protein activity. J Biol Chem 278, 46506-46515.

Hartwell, L. H., Culotti, J., Pringle, J. R., and Reid, B. J. (1974). Genetic control of the cell division cycle in yeast. 736 Science $183,46-51$.

Longtine, M. S., McKenzie, A., Demarini, D. J., Shah, N. G., Wach, A., Brachat, A., Philippsen, P., and Pringle, J.

740 Marshall, C. J. (1995). Specificity of Receptor Tyrosine Kinase Signaling - Transient Versus Sustained Extracellular 741 Signal-Regulated Kinase Activation. Cell 80, 179-185.

742 McKinney, J. D., Chang, F., Heintz, N., and Cross, F. R. (1993). Negative regulation of FAR1 at the Start of the 743 yeast cell cycle. Genes \& Development 7, 833-843.

744 Meloche, S., and Pouysségur, J. (2007). The ERK1/2 mitogen-activated protein kinase pathway as a master regulator 745 of the G1- to S-phase transition. Oncogene 26,3227-3239.

746 Mohl, D. A., Huddleston, M. J., Collingwood, T. S., Annan, R. S., and Deshaies, R. J. (2009). Dbf2-Mob1 drives 747 relocalization of protein phosphatase Cdc14 to the cytoplasm during exit from mitosis. J Cell Biol 184, 527-539. 
Nagiec, M. J., and Dohlman, H. G. (2012). Checkpoints in a yeast differentiation pathway coordinate signaling during hyperosmotic stress. PLoS Genetics 8 , e1002437.

750 Nagiec, M. J., McCarter, P. C., Kelley, J. B., Dixit, G., Elston, T. C., and Dohlman, H. G. (2015). Signal inhibition by a dynamically regulated pool of monophosphorylated MAPK. Mol Biol Cell 26, 3359-3371.

Oda, Y., Huang, K., Cross, F. R., Cowburn, D., and Chait, B. T. (1999). Accurate quantitation of protein expression and site-specific phosphorylation. Proc Natl Acad Sci USA 96, 6591-6596.

Oehlen, L. J., and Cross, F. R. (1994). G1 cyclins CLN1 and CLN2 repress the mating factor response pathway at

Oehlen, L. J., and Cross, F. R. (1998). Potential regulation of Ste20 function by the Cln1-Cdc28 and Cln2-Cdc28 cyclin-dependent protein kinases. J Biol Chem 273, 25089-25097.

Oehlen, L. J., Jeoung, D. I., and Cross, F. R. (1998). Cyclin-specific START events and the G1-phase specificity of

Oehlen, L. J., McKinney, J. D., and Cross, F. R. (1996). Ste12 and Mcm1 regulate cell cycle-dependent transcription

761 of FAR1. Mol Cell Biol 16, 2830-2837.

Orford, K. W., and Scadden, D. T. (2008). Deconstructing stem cell self-renewal: genetic insights into cell-cycle

764 Pelet, S. (2017). Nuclear relocation of Kss1 contributes to the specificity of the mating response. Sci. Rep. 7, 43636.

Pelet, S., Dechant, R., Lee, S. S., van Drogen, F., and Peter, M. (2012). An integrated image analysis platform to quantify signal transduction in single cells. Integrative Biology : Quantitative Biosciences From Nano to Macro 4, $1274-1282$.

768 Peter, M., and Herskowitz, I. (1994). Direct inhibition of the yeast cyclin-dependent kinase Cdc28-Cln by Far1.

769 Science $265,1228-1231$.

770 Peter, M., Gartner, A., Horecka, J., Ammerer, G., and Herskowitz, I. (1993). FAR1 links the signal transduction 771 pathway to the cell cycle machinery in yeast. Cell $73,747-760$.

772 Pumiglia, K. M., and Decker, S. J. (1997). Cell cycle arrest mediated by the MEK/mitogen-activated protein kinase 773 pathway. Proc Natl Acad Sci USA 94, 448-452.

Reményi, A., Good, M. C., Bhattacharyya, R. P., and Lim, W. A. (2005). The role of docking interactions in mediating signaling input, output, and discrimination in the yeast MAPK network. Mol Cell 20, 951-962.

Repetto, M. V., Winters, M. J., Bush, A., Reiter, W., Hollenstein, D. M., Ammerer, G., Pryciak, P. M., and Colman-

Roux, P. P., and Blenis, J. (2004). ERK and p38 MAPK-Activated Protein Kinases: a Family of Protein Kinases

780 with Diverse Biological Functions. Microbiology and Molecular Biology Reviews 68, 320-344.

781 Roy, A., Lu, C. F., Marykwas, D. L., Lipke, P. N., and Kurjan, J. (1991). The Aga1 Product Is Involved in Cell-

782 Surface Attachment of the Saccharomyces-Cerevisiae Cell-Adhesion Glycoprotein a-Agglutinin. Mol Cell Biol 11, 783 4196-4206.

784 Saito, H. (2010). Regulation of cross-talk in yeast MAPK signaling pathways. Current Opinion in Microbiology 13, $785 \quad 677-683$.

786 Sharifian, H. et al. (2015). Parallel feedback loops control the basal activity of the HOG MAPK signaling cascade. 
Integrative Biology : Quantitative Biosciences From Nano to Macro 7, 412-422.

Shou, W. Y., Seol, J. H., Shevchenko, A., Baskerville, C., Moazed, D., Chen, Z., Jang, J., Charbonneau, H., and from nucleolar RENT complex. Cell 97, 233-244.

791 Strickfaden, S. C., Winters, M. J., Ben-Ari, G., Lamson, R. E., Tyers, M., and Pryciak, P. M. (2007). A mechanism

792 for cell-cycle regulation of MAP kinase signaling in a yeast differentiation pathway. Cell 128, 519-531.

793 Tyers, M. (1996). The cyclin-dependent kinase inhibitor p40SIC1 imposes the requirement for Cln G1 cyclin

794 function at Start. Proc Natl Acad Sci USA 93, 7772-7776.

795 Tyers, M., and Futcher, B. (1993). Far1 and Fus3 link the mating pheromone signal transduction pathway to three

796 G1-phase Cdc28 kinase complexes. Mol Cell Biol 13, 5659-5669.

797 Visintin, R., Hwang, E. S., and Amon, A. (1999). Cfil prevents premature exit from mitosis by anchoring Cdc14

798 phosphatase in the nucleolus. Nature $398,818-823$.

799 Wassmann, K., and Ammerer, G. (1997). Overexpression of the G1-cyclin gene CLN2 represses the mating pathway

800 in Saccharomyces cerevisiae at the level of the MEKK Ste11. J Biol Chem 272, 13180-13188.

801 Wosika, V., Durandau, E., Varidel, C., Aymoz, D., Schmitt, M., and Pelet, S. (2016). New families of single 802 integration vectors and gene tagging plasmids for genetic manipulations in budding yeast. Molecular Genetics and 803 Genomics 291, 2231-2240.

804 Wu, C., Leeuw, T., Leberer, E., Thomas, D. Y., and Whiteway, M. (1998). Cell cycle- and Cln2p-Cdc28p-

805 dependent phosphorylation of the yeast Ste20p protein kinase. J Biol Chem 273, 28107-28115.

806 Yu, R. C., Pesce, C. G., Colman-Lerner, A., Lok, L., Pincus, D., Serra, E., Holl, M., Benjamin, K., Gordon, A., and

807 Brent, R. (2008). Negative feedback that improves information transmission in yeast signaling. Nature 456, 755-

808761.

809

810 Supplementary Material:

811

812 Supporting Information Legends

813 Supplementary Figures 1 to 11

814 Supplementary Tables 1 and 2 
bioRxiv preprint doi: https://doi.org/10.1101/274787; this version posted May 1, 2019. The copyright holder for this preprint (which was not certified by peer review) is the author/funder, who has granted bioRxiv a license to display the preprint in perpetuity. It is made available under

A

- Pheromone+ Pheromone
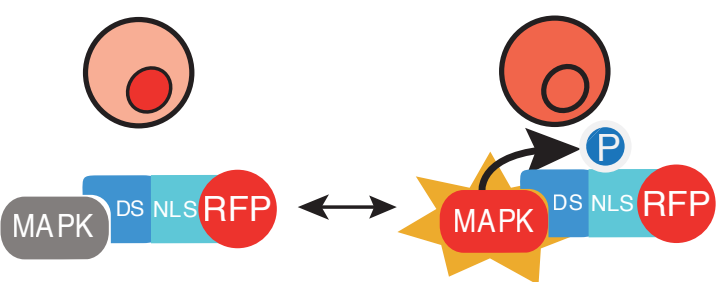

B

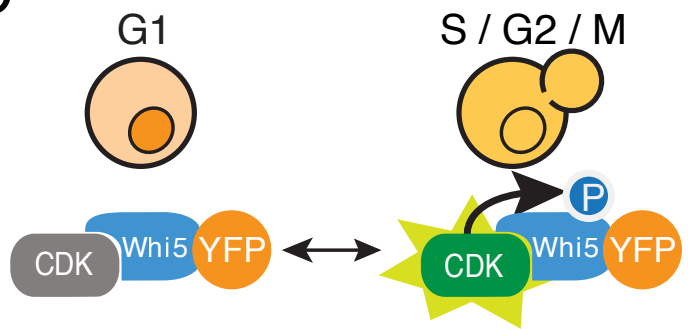

MAPK DS NLS RFP

$\mathrm{E}$
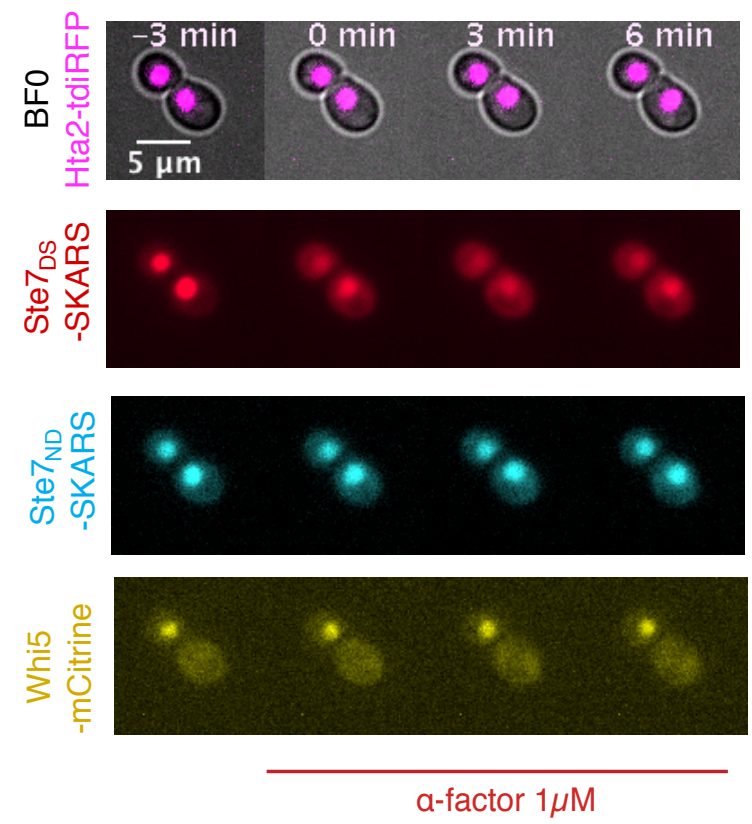

C

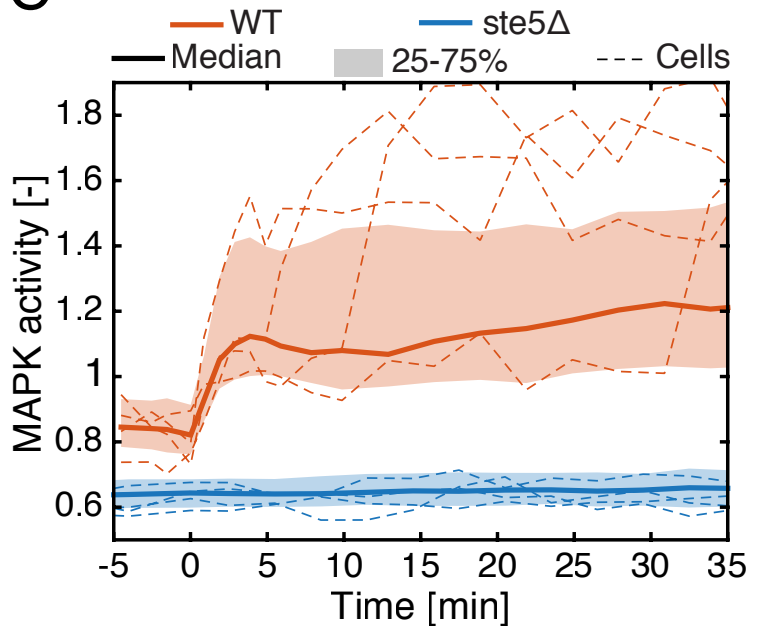

F



Figure 1 
bioRxiv preprint doi: https://doi.org/10.1101/274787; this version posted May 1, 2019. The copyright holder for this preprint (which was not certified by peer review) is the author/funder, who has granted bioRxiv a license to display the preprint in perpetuity. It is made available under

A aCC-BY 4.0 Intergational license.

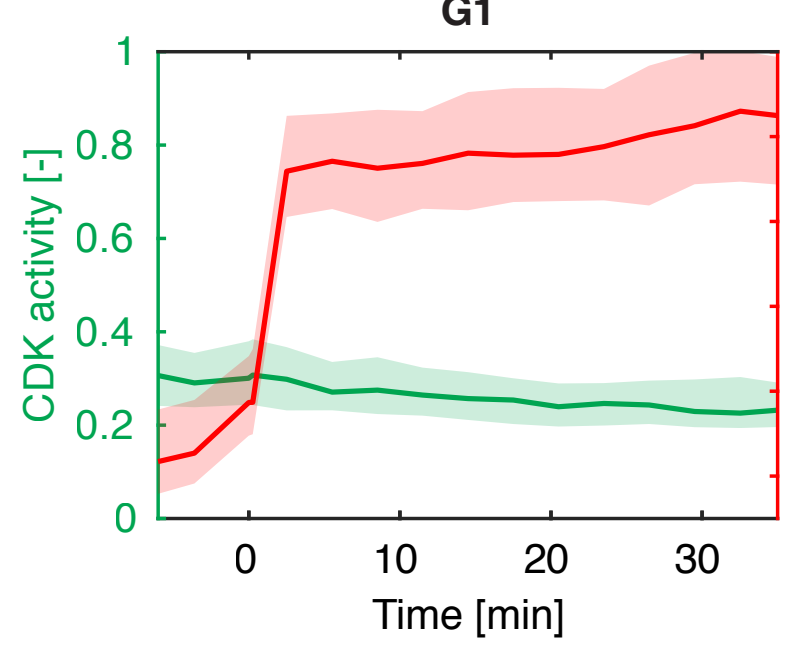

C
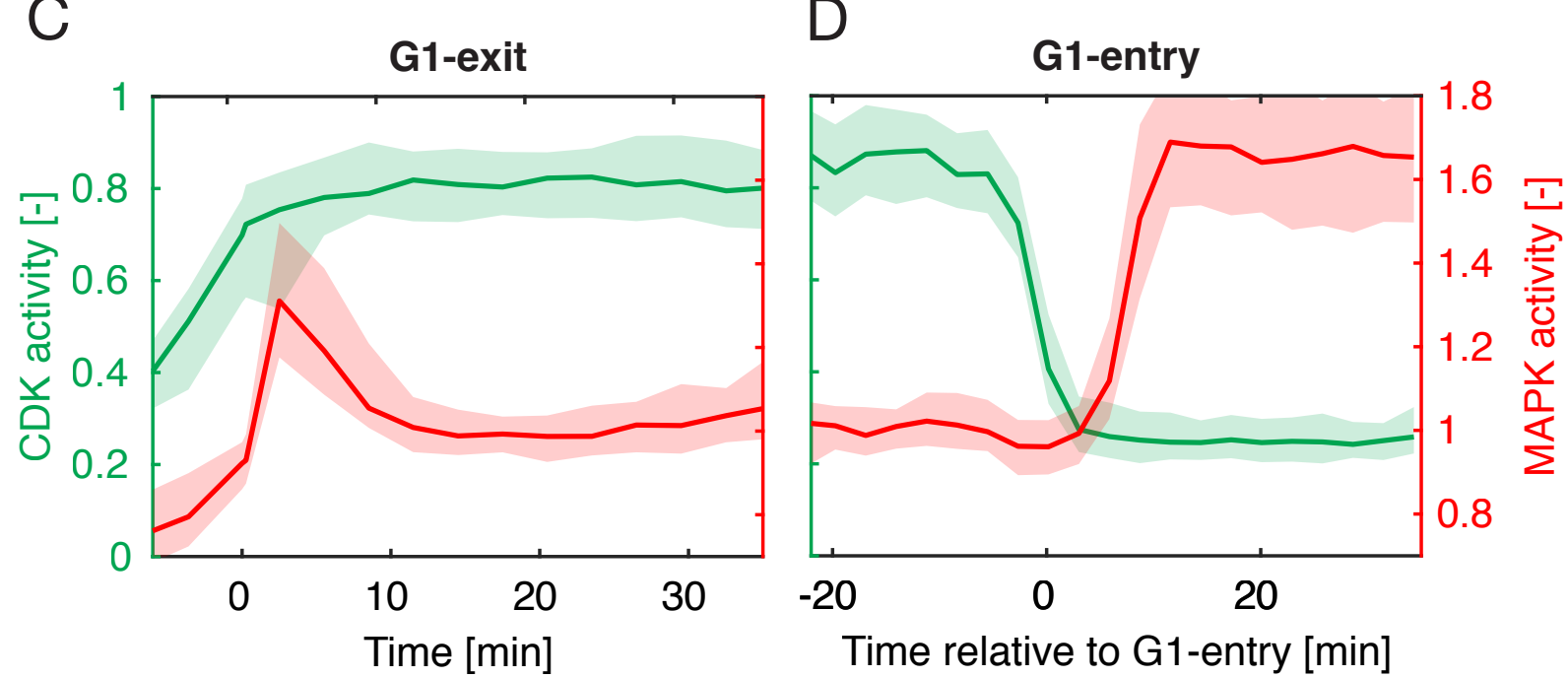

E - Out-of-G135\% - G1-entry 31\%
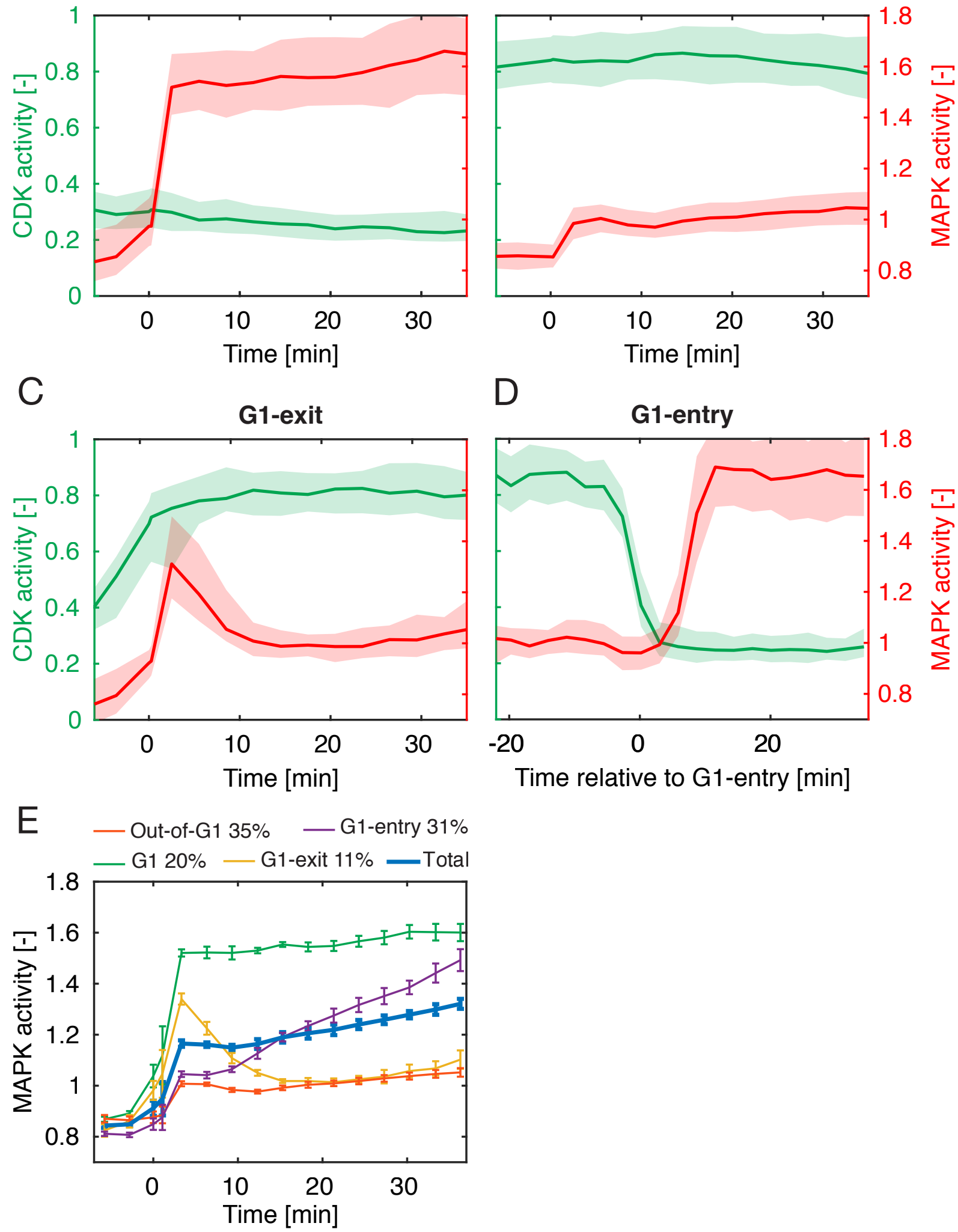

Time relative to G1-entry [min]

Out-of-G1 
bioRxiv preprint doi: https://doi.org/10.1101/274787; this version posted May 1, 2019. The copyright holder for this preprint (which was not certified by peer review) is the author/funder, who has granted bioRxiv a license to display the preprint in perpetuity. It is made available under aCC-BY 4.0 International license.

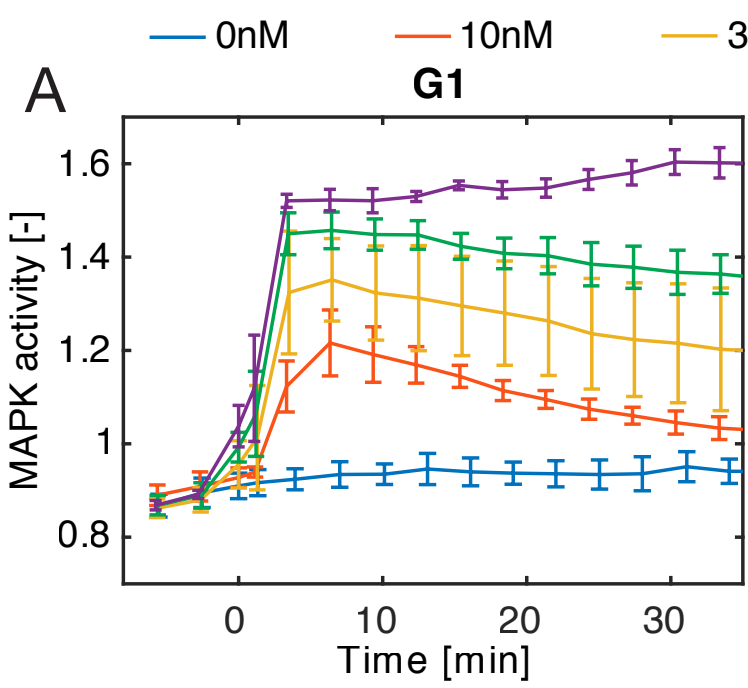

30nM

$-100 \mathrm{nM}$ 1000nM
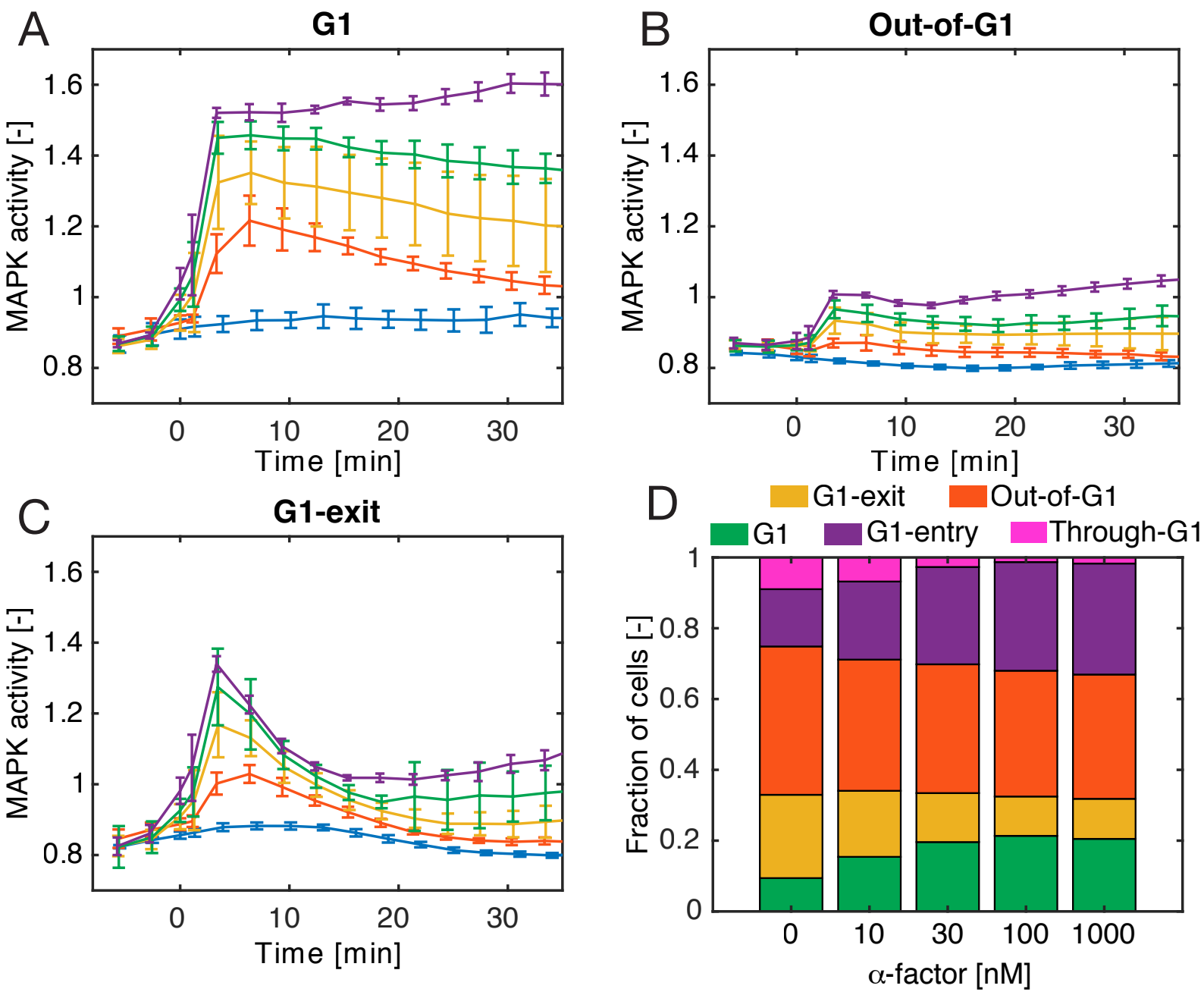

Figure 3 
bioRxiv preprint doi: https://doi.org/10.1101/274787; this version posted May 1, 2019. The copyright holder for this preprint (which was not

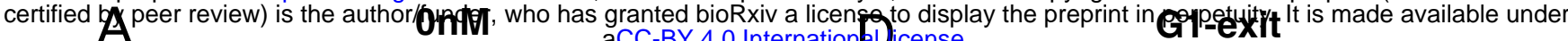
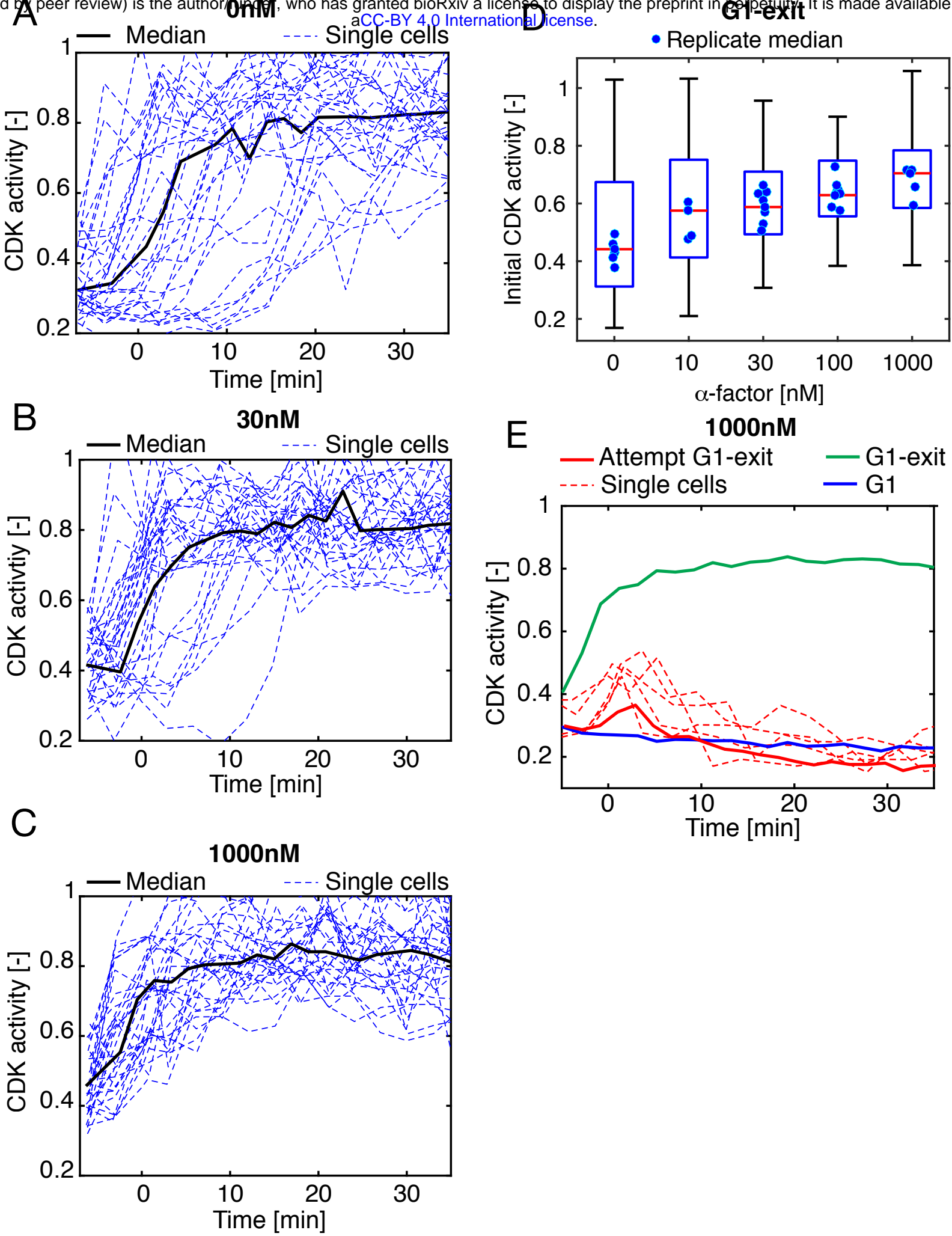

Figure 4 
bioRxiv preprint doi: https://doi.org/10.1101/274787; this version posted May 1, 2019. The copyright holder for this preprint (which was not certified by peer review) is the author/funder, who has granted bioRxiv a license to display the preprint in perpetuity. It is made available under A Start out of G1 aCC-BY 4.0 intern Bnal license.Attempt G1-exit
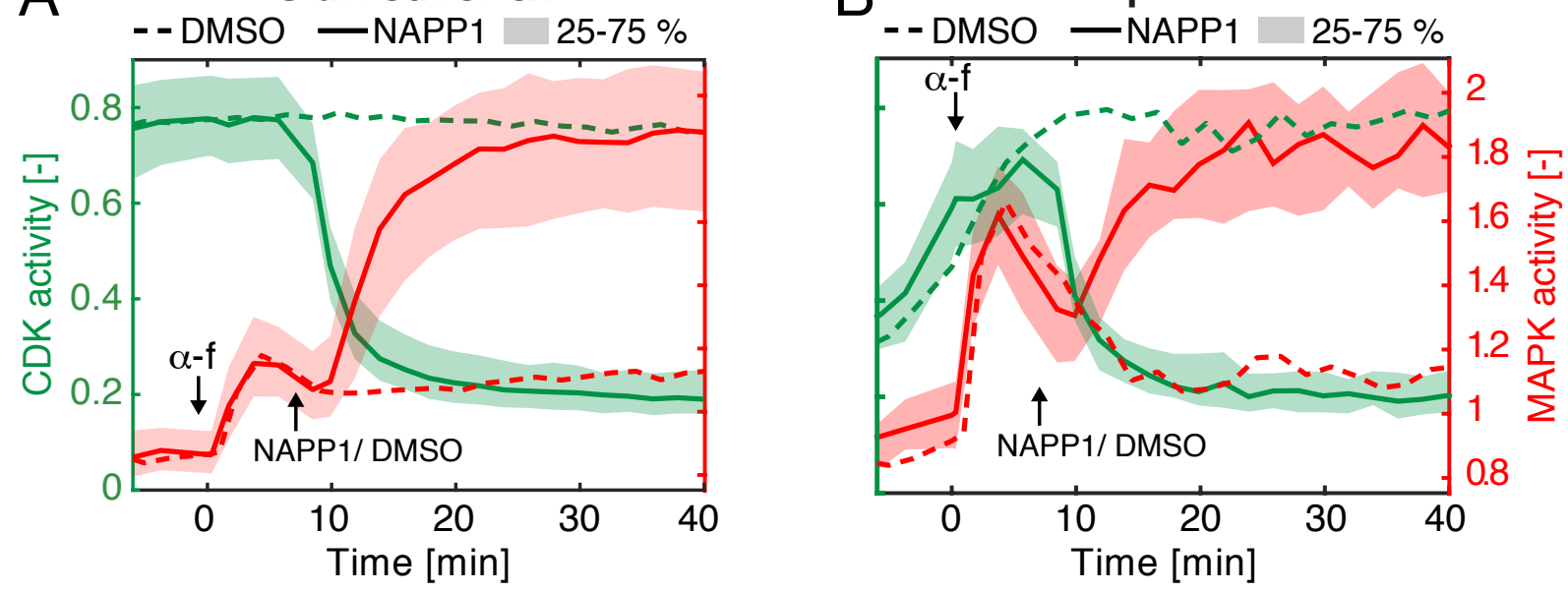

Figure 5 
bioRxiv preprint doi: https://doi.org/10.1101/274787; this version posted May 1, 2019. The copyright holder for this preprint (which was not certified by peer review) is the author/funder, who has granted bioRxiv a license to display the preprint in perpetuity. It is made available under

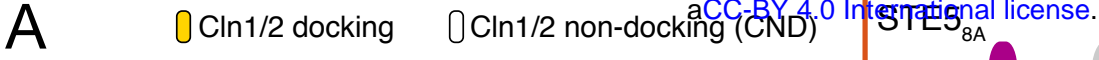

Plasma mb.
domain $\begin{gathered}\text { Interaction } \\ \text { domain }\end{gathered} \underset{\text { site }}{\text { Phosphorylation }}$

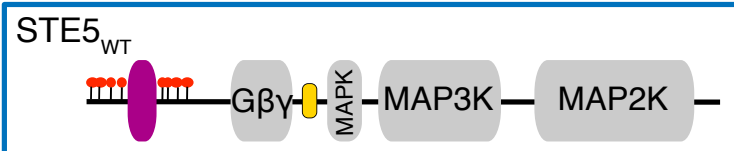

B

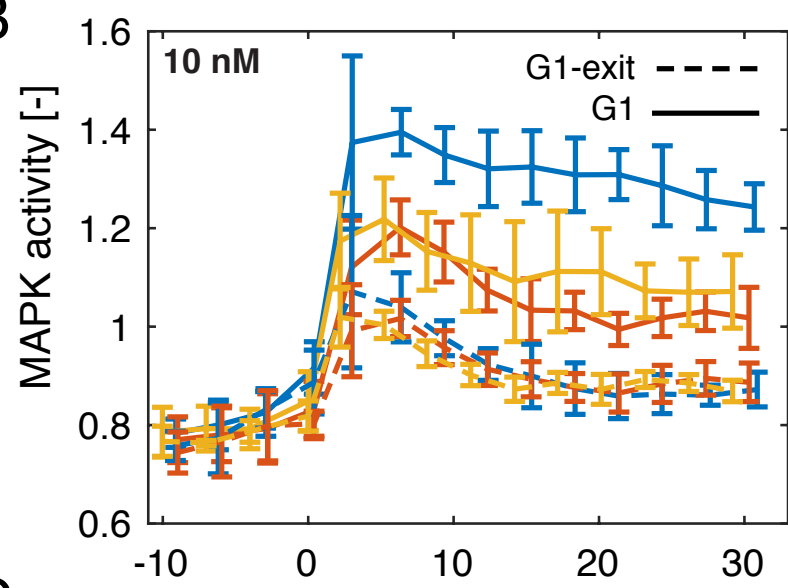

C
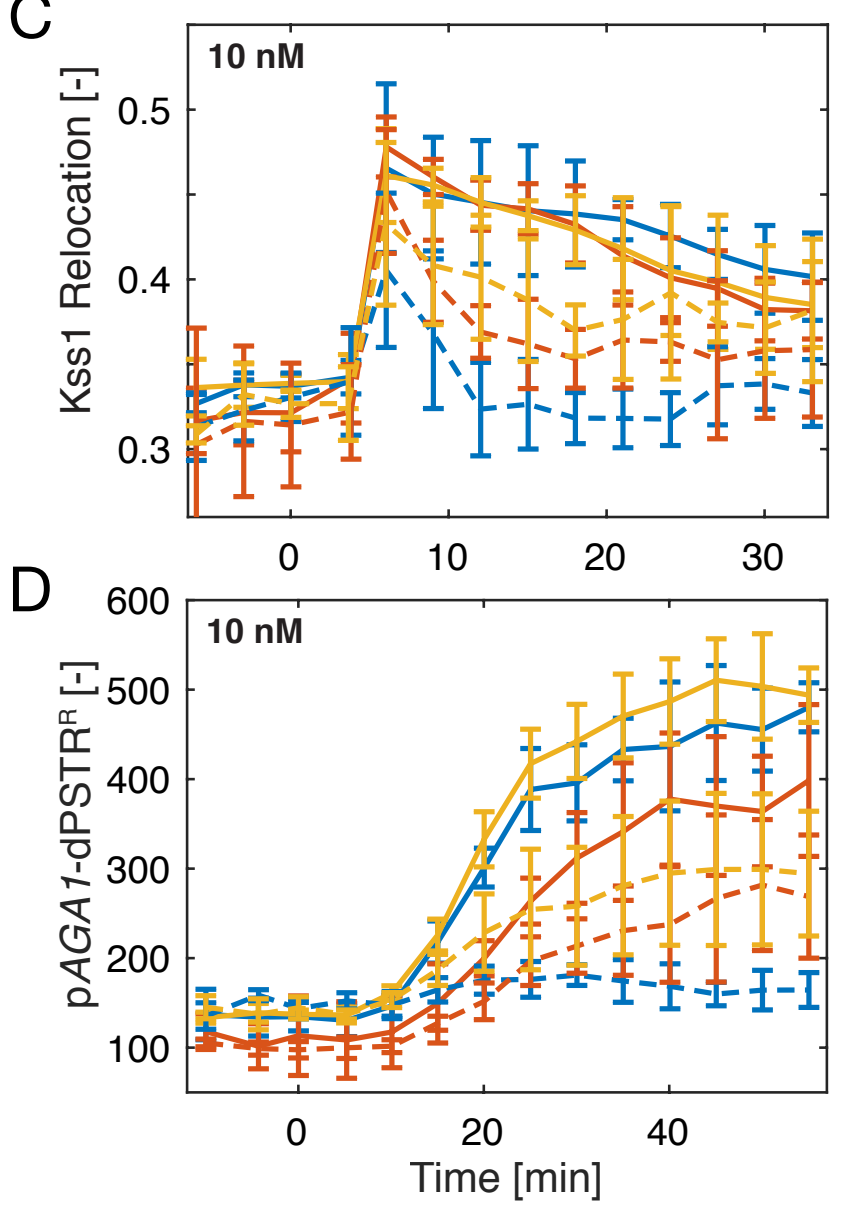

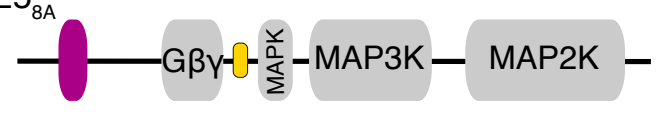

STE5 $_{\text {CND }}$
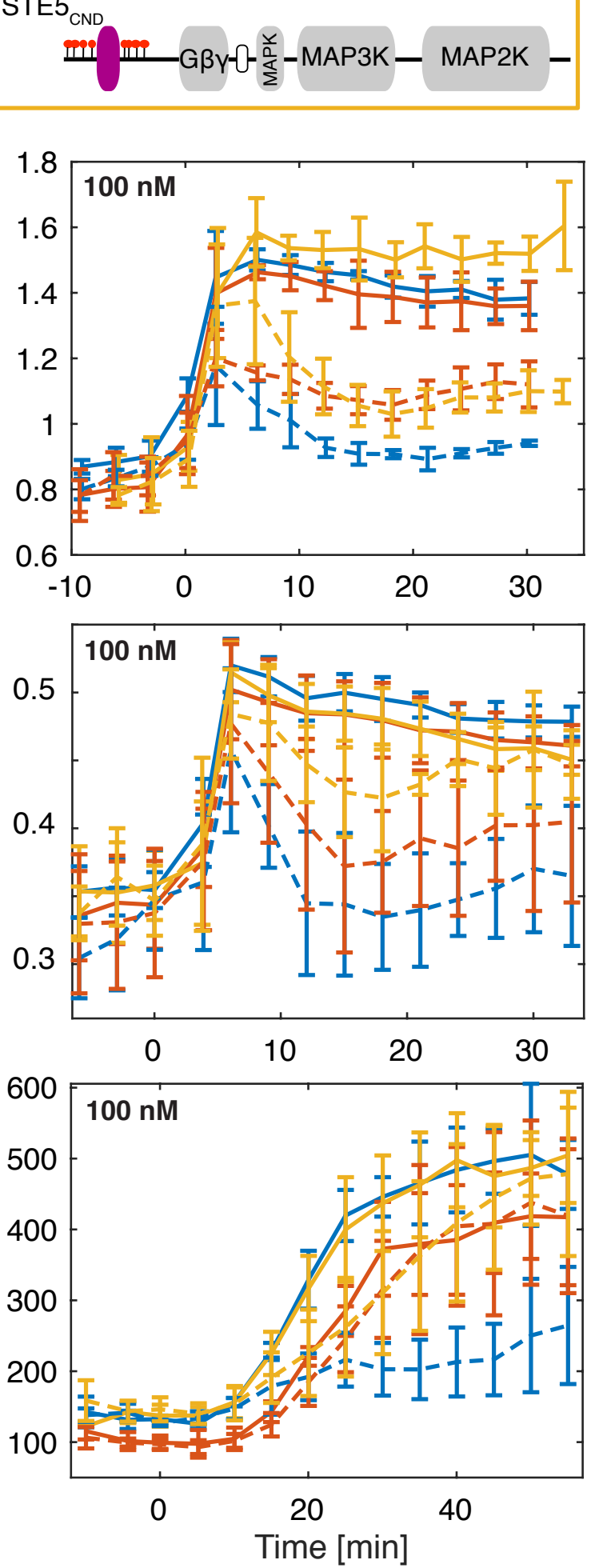
bioRxiv preprint doi: https://doi.org/10.1101/274787; this version posted May 1, 2019. The copyright holder for this preprint (which was not certified by peer review) is the author/funder, who has granted bioRxiv a license to display the preprint in perpetuity. It is made available under

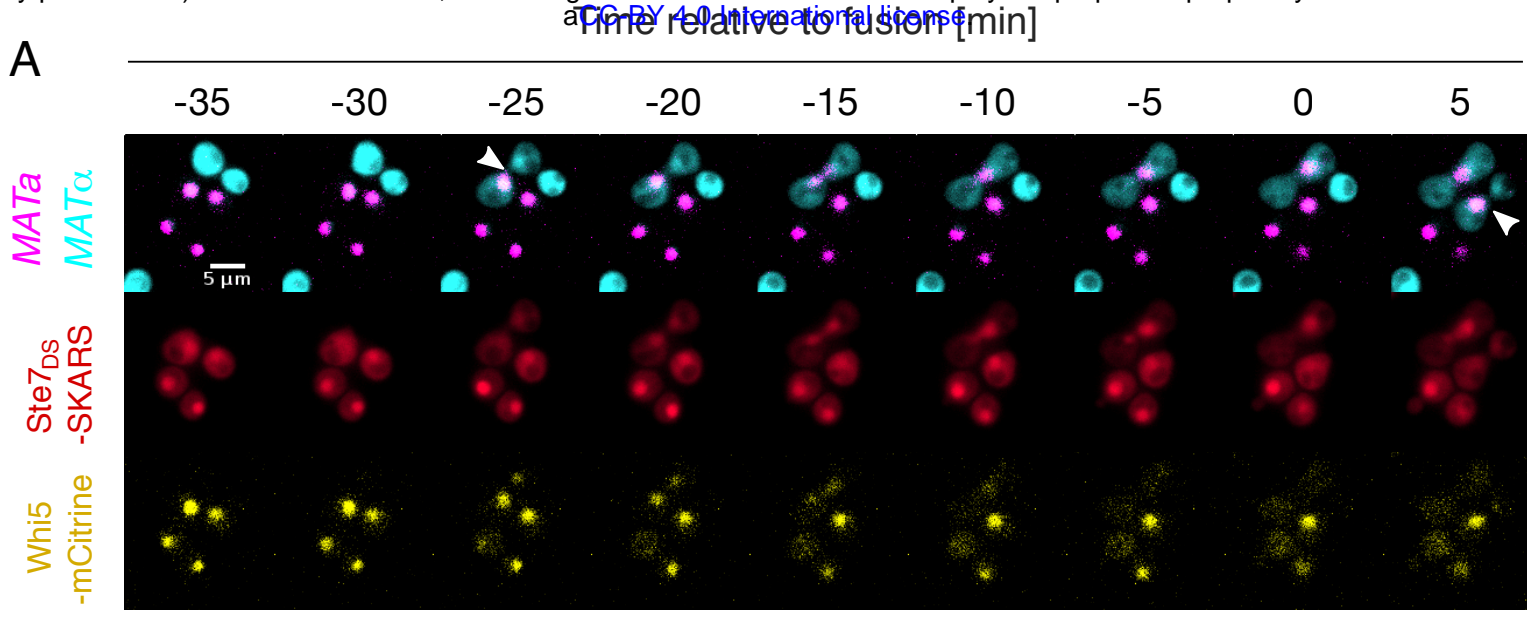

B

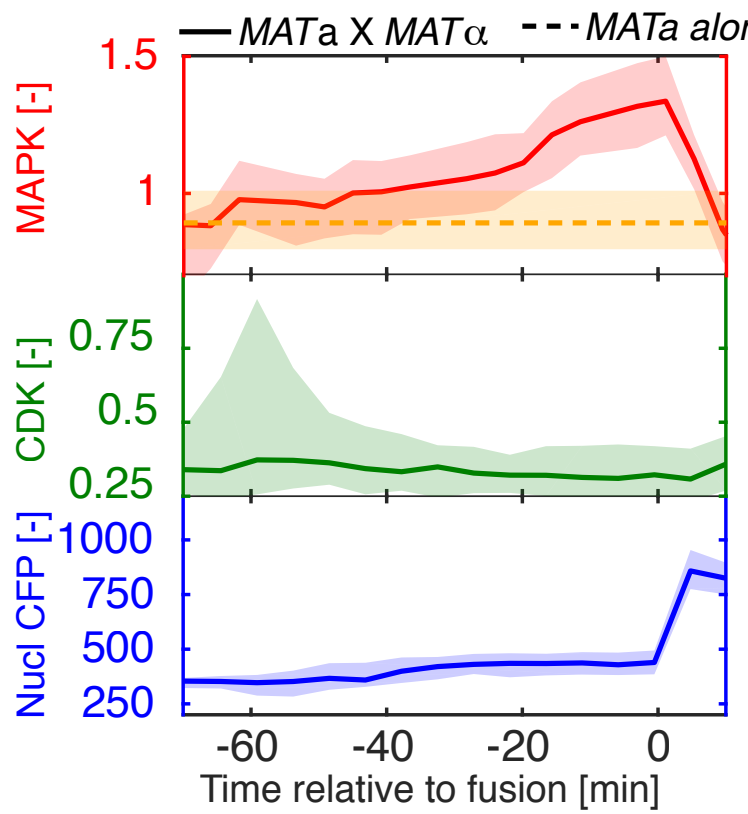

C

Mean $\Delta t$
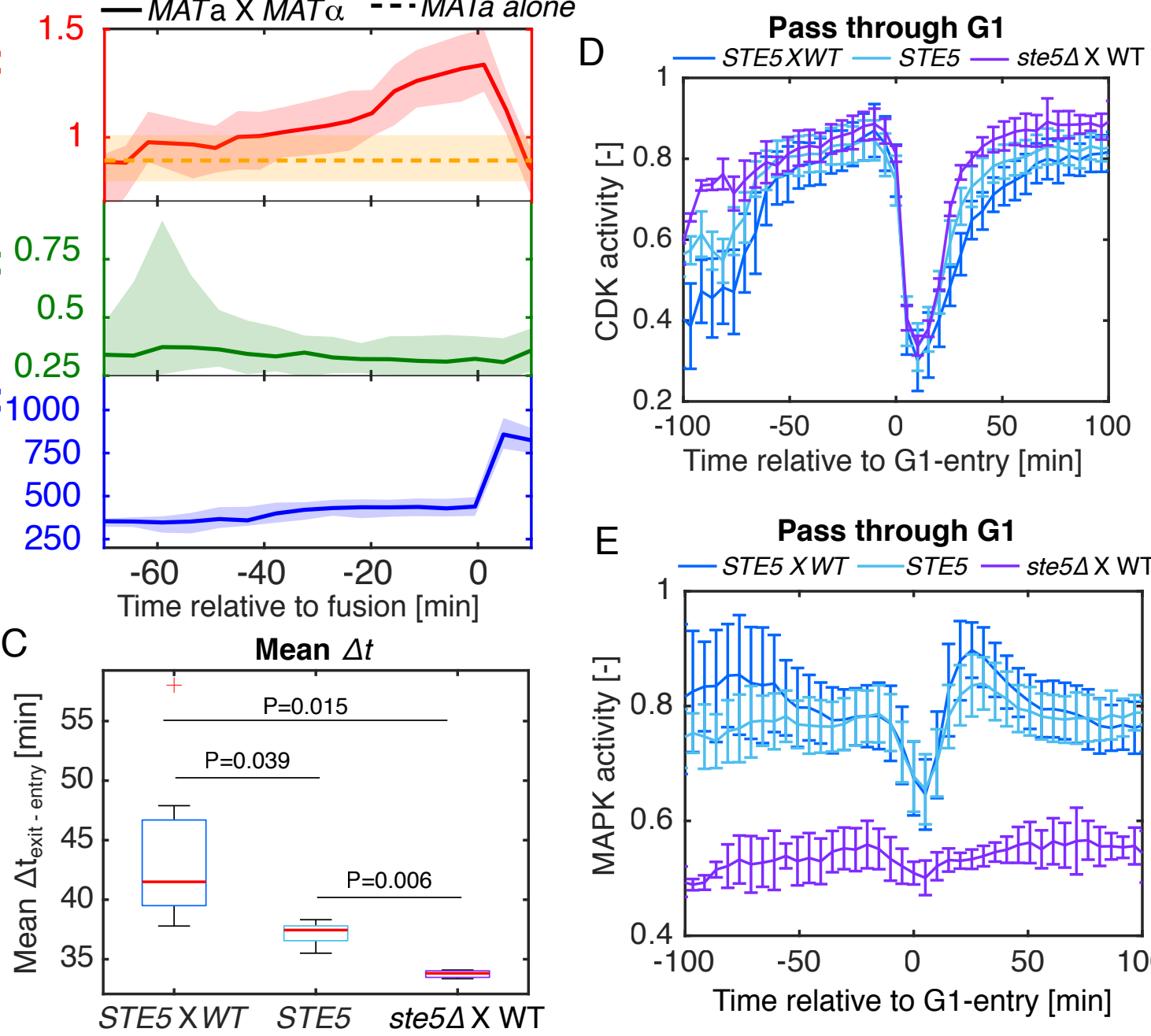

E Pass through G1

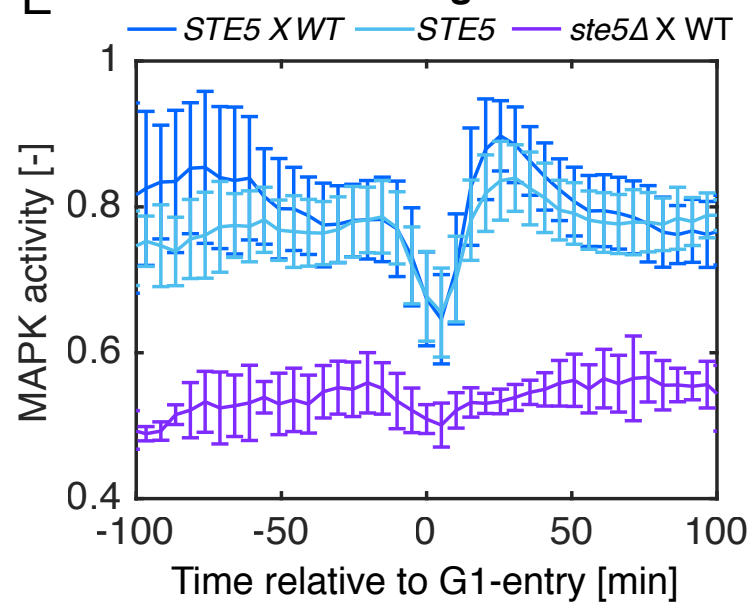


bioRxiv preprint doi: https://doi.org/10.1101/274787; this version posted May 1, 2019. The copyright holder for this preprint (which was not certified by peer review) is the author/funder, who has granted bioRxiv a license to display the preprint in perpetuity. It is made available under aCC-BY 4.0 International license.
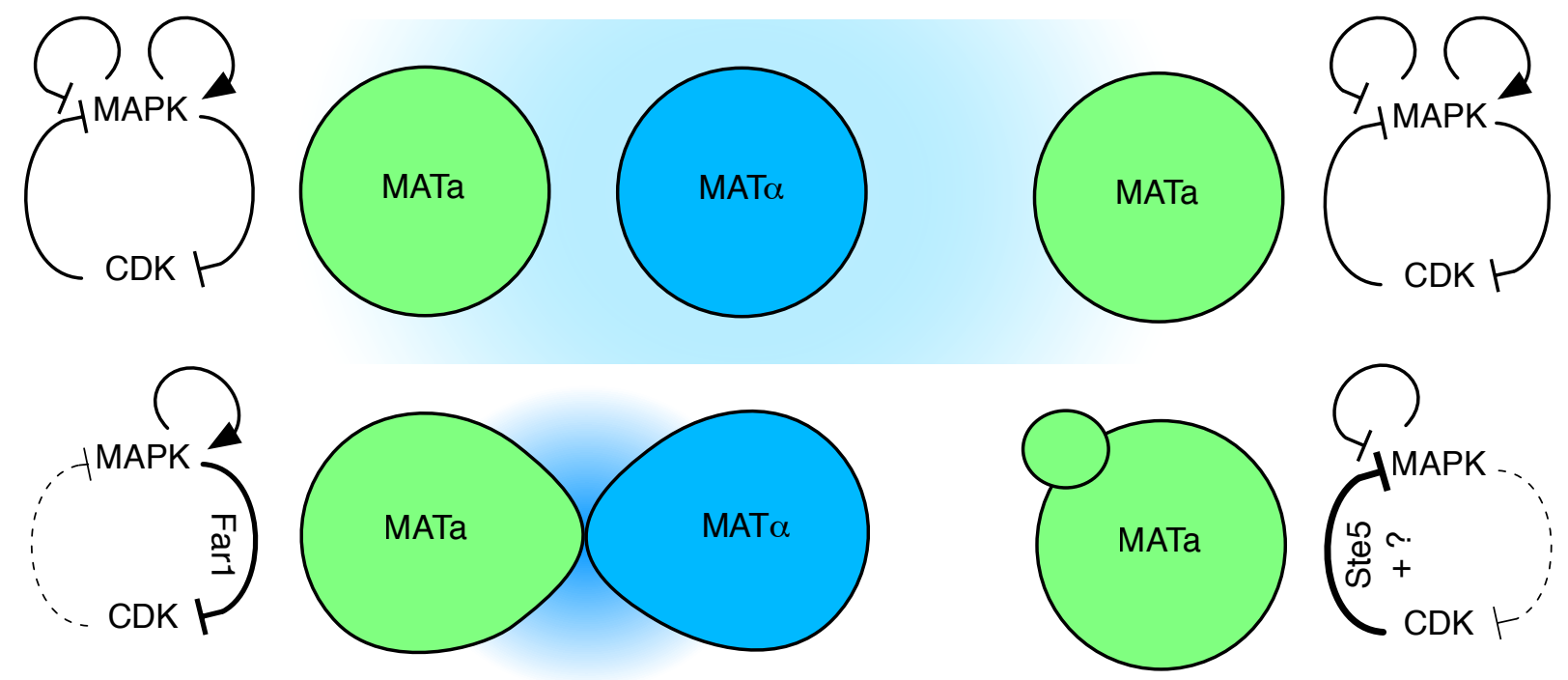

Figure 8 